\title{
Studies of Relativistic Heavy Ion Collisions at the AGS (Experiment 814)
}

W. E. Cleland

\section{University of Pittsburgh}

Department of Physics and Astronomy

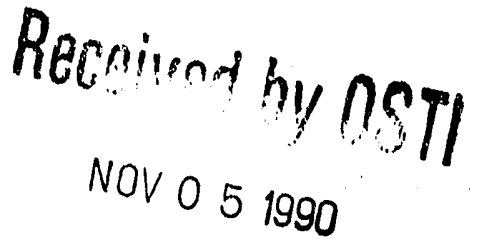

\author{
Annual Progress Report
}

DOE Grant No. DE FG02 87ER40363

1 May 1990 - 30 April 1991

\section{DISCLAIMER}

\begin{abstract}
This report was prepared as an account of work sponsored by an agency of the United States Government. Neither the United States Government nor any agency thereof, nor any of their employees, makes any warranty, express or implied, or assumes any legal liability or responsibility for the accuracy, completeness, or usefulness of any information, apparatus, product, or process disclosed, or represents that its use would not infringe privately owned rights. Reference herein to any specific commercial product, process, or service by trade name, trademark, ence herein to any specific commercial product, process, or service by trade name, traderecommanufacturer, or otherwise does not necessarily constitute or imply its endorsement, The views mendation, or favoring by the United States Government or any agency therect those of the and opinions of authors expressed herein do not
\end{abstract}


1. Introduction

\section{TABLE OF CONTENTS}

2. Status of the Experiment

2.1. Detectors

2.2. Physics results

2.2.1. Central collisions

2.2.2. Peripheral collisions

2.2.3. Search for exotic objects

3. Pitt Contributions to Experiment 814

3.1. Trigger Electronics

3.2. Participant Calorimeter

3.3. Multiplicity Counter

3.4. Forward Scintillation Counters

3.4.1. Performance Criteria

3.4.2. Electronics Chain

3.4.3. Z Identification for peripheral events

3.5. Beam Vertex Detector

3.6. Energy resolution in $\mathrm{Ur} / \mathrm{Cu}$ calorimeters

4. Publications

5. Personnel 


\section{LIST OF FIGURES}

Figure 2-1: Diagram of the apparatus of Experiment 814. M1 and M2 are dipole magnets. DC1. DC2, and DC3 are drift chambers used to track particles in the forward spectrometer. Solid lines in the figure represent trajectories for neutral particles, beamlike particles (with $\mathrm{Z} / \mathrm{A}=1 / 2$ ) and protons with $14.6 \mathrm{GeV} / \mathrm{c}$ momentum.

Figure 3-1: Results of radioactive source scan on one of the towers of the participant calorimeter. The current in a PM tube is digitized (shown here as the ordinate) as the source is drawn past the scintillator tiles. The abscissa is the position of the source, as measured in an optical encoder driven by the source transport system. The peaks correspond to individual scintillator tiles. The top two plots are for the EM sections. and the lower two plots are for the hadronic sections.

Figure 3-2: Data from the EM2 section of the tower shown in Figure 3-1 in which a fit for the optical coupling constants has been made. An exponential fallof $f$ has been included to account for contribution of the source to the current when it is beyond the actual scintillator plate. The amplitudes of the peaks are proportional to the optical coupling strengths. and these values are fed into the EGS Monte Carlo to simulate the response of the calorimeter.

Figure 3-3: Fraction of the energy seen in EM2 for seven of the calibration towers at two different electron beam energies. The Monte Carlo calculations include the optical constant obtain from the fits of the type shown in Figure 3-2. The tower-to-tower variations are clearly explained by the differences in optical coupling constants.

Figure 3-4: Histogram of the difference between the measured weights and the target weights in the summing circuits for the participant calorimeter.

Figure 3-5: An axonometric drawing of the two detectors

Figure 3-6: $\quad$ Measured $d \sigma / d n$ for $\mathrm{Si}$ beam with $\mathrm{Pb}, \mathrm{Cu}$ and $\mathrm{Al}$ targets from June 21 1989 run

Figure 3-7: Test of dead pad and multiple occupancy correction to $d \sigma / d n$ curve using FRITIOF ( $\mathrm{Si}$ beam on $\mathrm{Pb}$ target)

Figure 3-8: $\quad d n / d \eta$ plots for $\mathrm{Si}$ beam on (a) $\mathrm{Al}$ (b) $\mathrm{Cu}$ and (c) $\mathrm{Pb}$ targets for three different multiplicity triggers (June 1989)

Figure 3-9: $\quad d n / d \eta$ plots for $\mathrm{Si}$ beam on (a) $\mathrm{Al}$ (b) $\mathrm{Cu}$ and (c) $\mathrm{Pb}$ targets for three 24 different multiplicity triggers (June 1990)

Figure 3-10: Parameters $a, b, k$ and $\langle n\rangle$ calculated from E814 multiplicity 25 distributions in different rapidity intervals. All intervals start from rapidity 1 and are not symmetric.

Figure 3-11: Multiplicity $(0.9<\pi<3.9)$ vs. $E_{T}$ measured in TCAL $(-0.5<\pi<0.8)$ (a) 27 triggered on $E_{T}$ and (b) triggered on multiplicity

Figure 3-12: Total energy in a $0.8^{\circ}$ cone measured by UCAL vs. multiplicity 28

Figure 3-13: Total $E_{T}$ measured by PCAL vs. multiplicity for (a) multiplicity > 10 , (b) multiplicity > 70. (c) and (d) are the corresponding (uncorrected) PCAL $E$, spectra. The data were taken with a $\mathrm{Pb}$ target.

Figure 3-14: Schematic of the E814 detector layout during June 1990 peripheral 33 run which were used for the charge measurements 
Figure 3-15: (a) Measured charge distribution for $\mathrm{Si}$ detector vs vertical counter 34 V20. (b) Same distribution with horizontal counter H3. Beam passes through both counters.

Figure 3-16: (a) Correlation between vertical and horizontal scintillator hodoscopes 36 after Birks parameterization (b) $Z$ spectrum after combined measurement (double charge measurement)

Figure 3-17: (a) Correlation between $\mathrm{Si}$ detector just after the target vs the double charge measurement (b) $\mathrm{Z}$ spectrum after double charge measurement with combining Si detector (triple charge measurement)

Figure 3-18: Drawing of the beam vertex detector (upper) and its location in the E814 experimental setup (lower).

Figure 3-19: Schematic diagram of the electronics used for the beam vertex 39 detector.

Figure 3-20: (a) Trajectories of beam particles determined by the beam vertex system, shown in the horizontal plane. (b) Same as (a) but applying the $\mathrm{S} 1$ and $\mathrm{S} 3$ veto conditions. (c) Dispersion of the beam horizontal position $(\mathrm{mm})$ as a function of the position along the beam line $(\mathrm{cm})$.

Figure 3-21: (a) beam track extroplated to DC1 versus measured position in DC1. (b) Distribution of beam particles in DC1. (c) Difference between the position measured in the beam vertex detector extrapolated to DC1. and the position measured in DC1.

Figure 3-22: (a) Beam angle (mrad) versus beam position $(\mathrm{cm})$ at the horizontal counter H3, located behind the vertical forward scintillators. (b) Position distribution of beam particles at H3. (c) Position distribution of beam particles at $\mathrm{H} 3$, corrected to eliminate the dependence on the beam angle.

Figure 3-23: $\quad Z$ value of the triple charge measurements vs corrected position in 43 the horizontal counter $\mathrm{H} 3$.

Figure 3-24: (a) Energy dependence on $x$ position. (b) Energy dependence on $x$ position after the correction. In these plots a module of type $B$ is situated at $-200 \mathrm{~cm}$ and modules of type $A$ at $-180 \mathrm{~cm}$ and $-220 \mathrm{~cm}$.

Figure 3-25: The $x$-dependence of the response of the Type $\mathrm{C}$ calorimeter. 45

Figure 3-26: (a) $x$-dependence on proton energy after correction for spatial 46 dependence. (b) Corrected proton energy distribution. 


\section{LIST OF TABLES}

Table 3-1: Comparison of the gain shifts measured using the radioactive source with those measured by the optical source over three different periods of the June 1990 run. 


\section{Introduction}

During the past year. the Pittsburgh group has continued to work with the E814 collaboration (BNL - CERN - LANL - McGill - New Mexico - Pitt - Stony Brook - Tel Aviv - Texas A\&M - Yale) in carrying out AGS Experiment 814. In May 1987. during the heavy ion run at like AGS, we mounted a test setup. which was used (a) to understand the behavior of the modified uranium/scintillator calorimeters, (b) to test the prototype forward scintillation counters. (c) to carry out a set of albedo measurements, and (d) to carry out a measurement of the $E_{T}$ spectrum for a $10 \mathrm{GeV} /$ nucleon beam incident upon a variety of nuclear targets. Preliminary results [1] from this run indicate that there is almost complete stopping of the

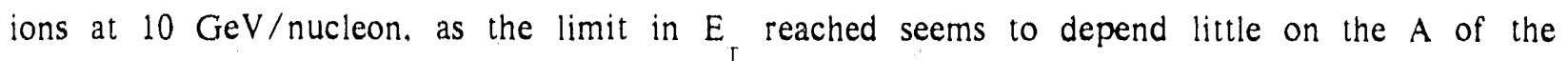
target nucleus. In November 1987, we mounted a smaller test in order to study the properties of scintillation counters in relativistic ion beams. In December 1988, the apparatus in its final configuration (but still incomplete) was exposed first to proton beams, in which debugging and testing was carried out, and then to $15 \mathrm{GeV} / \mathrm{c}^{28} \mathrm{Si}$ beams, during which a significant data sample was taken on each of the three major physics topics we are studying. These data have been analyzed. and three papers have been written for publication $[2,3,4]$. In June 1989. another run was carried out, in which all of the forward calorimeters were in place, while the drift chambers and the participant calorimeter were being tested. Results from this run have

been presented at various conferences $[5,6,7,8]$. Another ${ }^{28} \mathrm{Si}$ run was carried out in June 1990, in which the apparatus was finally complete, including the participant calorimeter, the drif 1 chambers, and a new device, the beam vertex detector. These data are still being analyzed.

As originally proposed. Experiment 814 is a study of both extreme peripheral collisions and the transition from peripheral to central collisions in relativistic heavy ion-nucleus interactions. It studies relativistic heavy ion interactions with nuclei in two types of collisions: (a) extreme 
peripheral collisions of large impact parameter, and (b) central collisions with high transverse energy in the final state. It emphasizes the measurement of overall event characteristics, in particular energy flow measurements and a precise measurement of the particle charge, momentum. and energy in the forward direction. This permits measurements of cross sections and rapidity densities as a function of the transverse energy for leading baryons emitted into regions of larger rapidity. Combining the energy flow measurements as a function of rapidity with the spectra of leading baryons provides information on the impact parameter dependence of the nuclear stopping of the projectile in relativistic heavy ion collisions. In 1988, the scope of Experiment 814 was enlarged to include a search for strange matter in central collisions. This idea. described in detail in a proposal submitted to the AGS [9], was initiated by J. Sandweiss and his Yale cowokers (R. Majka, F. Rotondo, and J. Slaughter). The basic physics argument behind the measurement is that large multiquark systems can be stable or metastable if three flavors of quarks are available, arising principally from the Pauli principle. The fact that the strange quark is heavy requires that the system have fairly large $A(A>10)$ to be stable. Simple models of the production of strangelets lead to the conclusion that they should be produced with a laboratory angle of about $80 \mathrm{mr}$ and with a rapidity equal to that of the center of mass of the heavy ion-target "cylinder" system. Thus there is a small but finite acceptance in the E814 apparatus. Because our detectors permit the simultaneous measurement of charge. time of flight, and energy, it is possible to determine the $Z$ and $A$ of candidate particles. 


\section{Status of the Experiment}

We give below a brief description of the status of the experiment, beginning with a brief description of the various detectors. followed by a summary of the physics results which have been obtained. Details on the parts of the experiment for which the Pitt group is responsible are given in Section 3.

\subsection{Detectors}

A diagram of the experiment is shown in Figure 2-1. We give a brief description of each of the detector systems below. More detailed information is available in our pr zvious annual reports and in the publications and conference reports of the experiment.

- Beam vertex detector. The beam vertex detector is a pair of silicon strip detectors used to locate the horizontal position and angle of the incoming beam particle. This system was installed before the June 1990 run. in order to improve the resolution in the center of mass quantities in certain exclusive peripheral interactions. E. M. Takagui is starting to study the properties of this detector, and her first results are given in Section 3.5 .

- Beam counters. The beam detectors serve to define the presence of a beam projectile. to establish the time of the collision, and to identify the charge of the projectile. The telescope consists of two counters in coincidence and two other counters, with a hole for the beam. in anticoincidence. The geometrical coincidence signifies that a track has crossed the target. A pulse height measurement from one of the counters is used to establish the charge of the projectile. and the timing of the other counter is used to establish its time of arrival. The problems posed by large dynamic range in making good amplitude and timing measurements for scintillation counters operating in a heavy ion beam are nontrivial. Tests on the suitability of various scintillators and phototubes were carried out in a heavy ion run during the fall of 1987. On the basis of these tests. NE102 was chosen as the scintillator material, Hammamatsu R2083 as the phototube for good 'timing, and EM 9954 as the tube for good charge resolution. In June 1988, the final counters were tested. With oxygen incident, the charge resolution was measured to be $5-10 \%$, and the timing resolution is better than 100 ps.

- Target. The target for E814 is a simple disk target with a remote positioning control. The rotating target assembly was built by the McGill group and was installed in the mount which supports the multiplicity counter. The disk has eight positions, so that up to seven targets plus an emply frame may be used at any one time. The complement of targets is changed for the different programs, as peripheral. central. and strangelet iunning require different materials and thicknesses. 


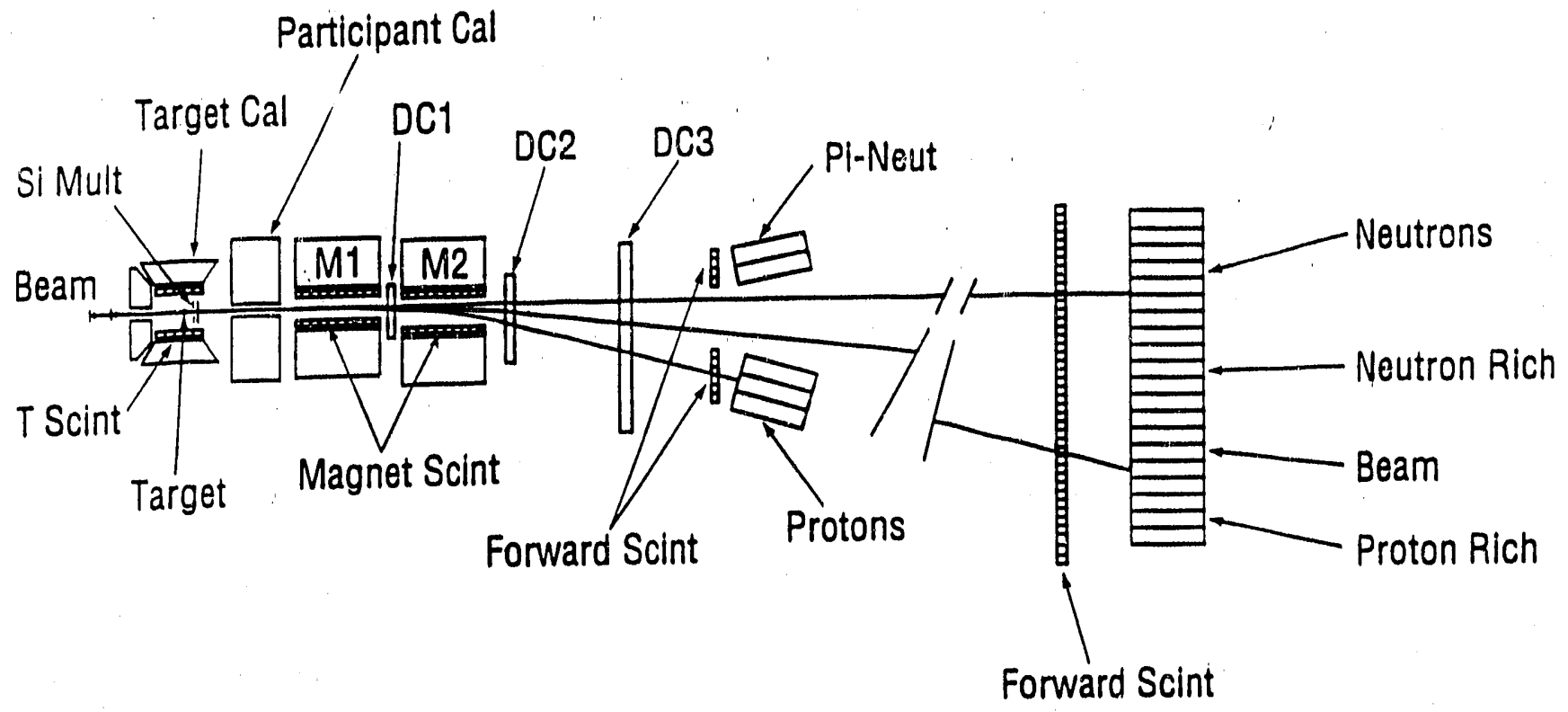

Figure 2-1: Diagram of the apparatus of Experiment 814. M1 and M2 are dipole magnets. DC1. DC2. and DC3 are drift chambers used to track particles in the forward spectrometer. Solid lines in the figure represent trajectories for neutra! particles. beamlike particles (with $\mathrm{Z} / \mathrm{A}=1 / 2$ ) and protons with $14.6 \mathrm{GeV} / \mathrm{c}$ momentum. 
- Multiplicity Detector. The silicon multiplicity counter consists of two 512 pad silicon detectors which are positioned immediately downstream of the target. They are used both at the trigger level and in offline studies of the multiplicity as a function of rapidity. This detector was fabricated at BNL, and the electronics was supplied by BNL. CERN, and New Mexico. K. Jayananda. a Pitt graduate student. was responsible for the pad layout and for designing the electronics for integrating it into the trigger. The detector was used successfully as a multiplicity trigger in the June 1989 run, and it was the source for most of the central triggers for the June 1990 run. Details of the detector and analysis of the multiplicity data are given in Section 3.3.

- Target Calorimeter. The target calorimeter is used to detect the products of the target fragmentation and covers the region of $\theta$ between $45^{\circ}$ and $180^{\circ}$. It consists of a box composed of 900 blocks of NaI, $4 \times 4 \mathrm{~cm}$ in cross section and 6 radiation lengths thick. It serves to measure $E_{r}$ in central collisions and it also acts as a veto for the peripheral collisions. The $\mathrm{NaI}$ is read out using photodiodes followed by preamplifiers and shaping amplifiers. Summing electronics for the first and second level trigger has been designed and fabricated at Stony. Brook. The construction and calibration of this detector is the responsibility of the Stony Brook group.

- Participant Calorimeter. The participant calorimeter, constructed by Los Alamos and Texas A\&M, is azimuthally symmetric, covering the polar angle region $1.0^{\circ}<\theta<$ $45^{\circ}$. It has both azimuthal and polar angle segmentation. The radiator is lead plates, and the sampling layer is plastic scintillator, coupled to phototubes via wavelength-shifting fiber light guides. The electromagnetic and hadronic sections of the calorimeter are read out separately. Fast trigger sums, using $E_{r}$ weights, are formed separately for each azimuthal section, both for the electromagnetic and hadronic sections of the calorimeter. Z. Zhang, a Pitt graduate student. is studying the problem of bringing the PCAL $E_{T}$ trigger to an operational state, including the problem of on-line gain monitoring and adjustment. A discussion of the status of the calorimeter is given in Section 3.2

- Tracking Chambers. In order to measure the momenta of the charged particles emitted in the collisions, we use a spectrometer consisting of three sets of tracking chambers and two magnets. This configuration is chosen due to the wide range of particle momenta. Particles with momenta greater than $6 \mathrm{GeV} / \mathrm{c}$ are measured in all three sets of tracking chambers, yielding a momentum accuracy of about $1 \%$, whereas particles of momenta less than $6 \mathrm{GeV} / \mathrm{c}$ are seen only in the first set of tracking chambers. giving only a crude measurement of the momentum. One of the chambers, DC1, has highly segmented pad readout and interpolation by charge division. It is positioned between the two magnets. The chamber has 1000 pads. and the density varies across the plane in accordance with the expected track multiplicity. The design of this chamber was carried out in the Instrumentation Division of BNL. One of the key people in this group is Bo $Y u$, a graduate student at the University of Pittsburgh. The other drift chambers for the experiment. DC2 
and DC3. consist of six drift planes and two planes with cathode pad readout. They have been designed at BNL, with the fabrication of the frames carried out at Yale. These chambers were installed just before the June 1989 run and were fully operational in the June 1990 run.

- Forward Calorimeters. Charged particles with momenta greater than $6 \mathrm{GeV} / \mathrm{c}$ and all neutral particles emitted in a cone of $\theta<1.0^{\circ}$ are measured in the forward calorimeters. In 1988, we had only 13 of these units, since they were obtained from CERN and were still required in HELIOS. The remaining 12 units were shipped to BNL in early 1989 and were modified and installed in the experiment before the June 1989 run, completing the set of uranium/scintillator calorimeters in the forward spectrometer. Just upstream of each of the calorimeter stacks are a bank of scintillation counters, two counters associated with each stack, which are ised to measure the charge and the vertical position of the track. Because fragments emitted in the forward direction have approximately the velocity of the beam particle ( 15 $\mathrm{GeV} /$ nucleon), the energy measurement in the calorimeters is a good measure of the nucleon number. The use of calorimeters in the detection of final state neutrons is particularly attractive, as the good energy and spatial resolution of these calorimeters permit studies of correlations among leading neutrons in order to get information on transparency in nuclear collisions and to search for bound multineutron states. Thus is is important for us to understand the detailed response of these units to the forward emitted baryons. Our graduate student. U. Sonnadara has been studying their properties (see Section 3.6).

- Scintillation Counters. There are three sets of scintillation hodoscopes used in the experiment: (a) the target counters, used in conjunction with the target calorimeter to identify charged particle fragments and neutrals: (b) magnet scintillators, which line the sidewalls of the two magnets, used to detect pions produced in the forward direction: and (c) forward scintillation counters, to be used in conjunction with the forward calorimeter to measure the charge of nuclear fragments. The target counters have been constructed and installed by the Stony Brook group. The magnet counters and forward scintillation counters have been constructed and installed by the Pitt group. and their operation and calibration is the responsibility of U. Sonnadara. The status of these counters is discussed more fully in 3.4.3.

\subsection{Physics results}

Experiment 814 has studied Si + A collisions in three running periods: December 1988. June 1989. and June 1990. Results from the 1988 run on central collisions [2], peripheral interactions [3], and the strangelet search [4] have been published. Data analysis from the 1989 run has been finished. and these results have been presented in Quark Matter '90 [8, 10] and at the HIPAGS workshop [5, 6, 7]. The 1990 data are still under study, and some of 
these efforts are described in Section 3. We give here a brief summary of some of the more important results of this work and indicate how it. will be extended in the future.

\subsubsection{Central collisions}

The main features of Experiment 814 of importance for centrai collision studies are (a) its ability to measure global event characteristics $E_{T}$ in the target and participant calorimeters and charged multiplicity in the silicon multiplicity detector), and (b) its ability to measure the $p_{T}$ spectra of forward emitted baryons at $0^{\circ}$. Because the two are combined in one experiment, it is possible to measure the dependence of these spectra on centrality of he collisions.

We have measured with this apparatus, $E_{T}$ spectra in the backward region, using the target calorimeter, and multiplicity spectra in the forward region. Both of these spectra can be reproduced using a version of Hijet with rescattering of the secondary particles. Other Monte Carlo codes which ignore this effect are unable to explain the large energy flow in the backward direction.

We have found that the probability for a baryon to pass through the target and retain its rapidity becomes essentially constant as a function of $E_{T}$ in the event, and that its mean free path is near that of unmodified nuclear matter. We have also measured the spectra of protons and neutrons at very low $\mathrm{p}_{T}$, where other experiments have seen anomalously large production rates. At the moment. comparison with data from other experiments is difficult, due to the different $p_{T}$ ranges and triggering conditions. We plan to extend the range of our measurements during the coming heavy ion run to alleviate this problem.

We include in this report (Appendix C) a more extended summary of E814 results on central collisions. prepared recently (September 90) for the BNL Program Advisory Committee. 


\subsubsection{Peripheral collisions}

The initial study of electromagnetic dissociation showed that the simple picture of a onephoton absorption, when modified with the low energy photonuclear crosis section. explained the one-nucleon EMD cross sections very well. For more complex final states, the inclusive cross section. can be well reproduced by calculations which include a folding of the Giant Dipole Strength function of the projectile, the virtual photon spectrum from line target and the statistical decay of the excited projectile. We are now extending these measurements in two directions: (1) the addition of drift chambers and the beam vertex detector will permit a reconstruction of the actual excitation energy spectrum, and (2) improvement in the trigger permits studies of lower cross section states, such as those containing 2 or 3 protons or neutrons in the final state. The statistical strength of the new sample is such that we may be able to observe the small effects of multiphoton excitations.

\subsubsection{Search for exotic objects}

With its long flight path. good tracking ability, and its total energy measurement through calorimetry, the forward spectrometer of experiment E814 is a unique facility to search for exotic objects such as strangelets. From a two day running period in December 1988. using a very simple non-selective trigger, we already set a limit on the production of strange matter with $\mathrm{Z} / \mathrm{M}$ in the range 0.1 to 0.3 of less than $1.2 \times 10^{-1}$ per interaction for multiply charged and less than $8.3 \times 10^{-4}$ per interaction for singly charged strangelets. In the June 1989 run, a time-of-flight trigger was introduced, and the resulting data will yield limits about a factor of ten higher than these values. Already at the QM'90 conference. with $1 / 6$ of the new data analyzed. the above upper limits are reduced $109.9 \times 10^{-5}$ and $3.4 \times 10^{-4}$. respectively. It will be possible. if we devote about 10 days to this program in the next run and tighten the trigger even more, to reach limits of about two orders of magnitude higher than those that will be set by the June 1989 data. 
Another area of exotic objects which can be detected in the E814 apparatus is pineuts, i.e. a multineutron state bounded to a $\pi^{-}$. Upper limits for the production cross sections of $\left(\pi^{-} 2 \mathrm{~N}\right)$, $\left(\pi^{-} 3 \mathrm{~N}\right)$, and $\left(\pi^{-} 4 \mathrm{~N}\right)$ production on targets of $\mathrm{Al}$. Sn. and $\mathrm{Pb}$ were presented at the QM 90 conference. Except for the $4 \mathrm{~N}$ states. for which our acceptance is low. the upper limits range from 0.2 to $14.2 \mu b$. 


\section{Pitt Contributions to Experiment 814}

The Pitt contributions to Experiment 814 involve activities in several detector systems: trigger electronics. the magnet and forward scintillators. the multiplicity counter. the beam vertex detector, and the participant and forward calorimeters. Each of these is discussed in the following sections.

\subsection{Trigger Electronics}

In general terms, the goals of the trigger are:

- to identify central collisions through a measurement of $E_{r}$ using the participant and target calorimeter:

- to identify extreme peripheral collisions through a measurement of energy above some threshold in the forward calorimeter and low $E_{T}$ elsewhere:

- to permit a selection of exotic (Z,A) values in the forward calorimeter at the trigger level.

- to permit a selection of events containing a slow particle in the final state, as measured via time of flight in the forward scintillators.

The system was designed to permit as much flexibility as possible through the use of softwarecontrolled logic. Flexible control is particularly important for this experiment, where so much is unknown in advance about the nature of the events. The approach is to create a sufficiently restrictive first level trigger such that a number of signals can be digitized in a fast-encoding $A D C$ system. and higher level triggers are created using digital processing of these signals.

The second level trigger processor is based on the use of FERAs which can produce digitization of an analog signal to 10-bit accuracy in $5.6 \mu \mathrm{s}$. These FERAs are read out at a frequency of $10 \mathrm{MHz}$ into ECL units, which are used to perform calculations for the trigger. Sor far, in this experiment, the following different types of calculations are performed:

1. Total $E_{T}$ sums for the target calorimeter. 
2. Total multiplicity sum for the multiplicity detector.

3. Charge and baryon numbers for each of the six sections of the forward calorimeter.

4. Time of flight, with slewing corrections. for the forward scintillators.

The methods used to carry out these calculations have heen presented in our previous annual reports.

In general, the trigger system has performed reliably, and the flexibility which was built into it from the beginning has been a useful feature. For example, adding the time-of-flight trigger, including a discriminator slewing correction, was rather easily accommodated. Trigger conditions for the selection of antiprotons, where calorimeter deposition can be correlated with the forward scintillators associated with each individual calorimeter has also been accommodated. The addition of the participant calorimeter. and if we wish. simultaneous inclusion of multiplicity and/or target calorimeter information with that from the participant calorimeter will be possible.

The Central Electronics Shop at Pitt was very helpful in carrying out the design and fabrication of a number of special purpose modules needed for this trigger system, and it still performs an important maintenance role in repairing any of these modules. In addition, this shop provides general maintenance for much of the rest of the electronics in the experiment. particularly for the large number of current monitor modules designed and built for this experiment.

We are also developing for E814, as a RHIC R\&D project. a trigger monitor workstation. based on artificial intelligence techniques. The first stage of this project. completed before the June 1990 run, was the development of an interrupt-driven data acquisition system which rescans the trigger CAMAC crates for each event. after the main data acquisition system has 
acquired the data. The workstation processes the trigger data. recalculating in sof tware the quantities" calculated by the hardware processors and compares the results with the trigger bit patterns generated in the hardware. In addition. the workstation has online histogramming possibilities, and since it samples $100 \%$ of the data. we are able to monitor the trigger of the experiment more thoroughly than was previously possible.

The software for the workstation was developed in such a way that the second stage of the project. namely the introduction of an expert system to help search for faults and to diagnose trigger problems, will be straightforward. Before we begin to create automatic diagnostic procedures. however, we are experimenting with a simpler "stand alone" diagnostic system, to be used to help experimenters unfamiliar with the trigger system to ask for help in case of a failure. This is an important development. since occasionally a system fails in such a way that data-taking is impossible. The program we are providing will be a step-by-step question and answer technique, which leads the experimenter through a series of checks to search for simple failures. such as failed power supplies. unplugged cables, switches incorrectly set. etc.. One of the objectives of this research is to see to what extent such a system will really be used by the non-experts. If it is found to be useful, we would plan to expand this technique to other systems in an experiment (such as OASIS) from the very beginning. This work is being carried out primarily by our graduate studient Z. Zhang. in collaboration with Dr. S. Clearwater of our Computer Science Department. A report of the systern was given at the International Workshop on Software Engineering, Artificial Intelligence and Expert Systems for High Energy and Nuclear Physics in Lyon France. March 1990 (see Appendix B). 


\subsection{Participant Calorimeter}

The Pitt group became involved in the participant calorimeter commissioning in the fall of 1989 with the formation of the PCAL lask force, chaired by W. Cleland. This group oversaw the commissioning of the PCAL, including replacement of the ADC system, revision of the source calibration system, installation of a DC optical source for gain stabilization. and substantial lowering of the gains of the photomultipliers. permitting uniform gain constants to be set over the entire calorimeter. The task force met through September 1990, and its report to the collaboration is included here as Appendix A.

Unlike many orher calorimeters, which use wavelength shifters as the optical coupling device. the participant calorimeter in Experiment 814 uses instead optical fibers for the coupling between the scintillator plates and the photomultiplier tubes. due to the special configuration of the spectrometer. This type of coupling is rather delicate, and good light coupling can be obtained with the proper care in construction. However, the bond can be broken with thermal and mechanical shocks. and the efficiency can then become essentially nonexistent. A careful study of the fiber coupling from time to time is crucial for understanding the performance of the calorimeter. As we show below, our radioactive source calibration gives valuable information of this type, and it is difficult to see how the performance of the calorimeter could be understood without it.

In the August 1990 collaboration meeting held at Stony Brook. Dan Fox presented a summary of the status of the PCAL. The new mode of operation of the radioactive source, in which the photomultiplier response from a slowly moving source is digitized in a precision current monitor. gives extremely detailed information concerning the fibe: optic coupling to the various plates in the calorimeter. In Fig. 3-1, we show an example of the current observed in the four different phototubes as the source is drawn past one of the towers. We see individual peaks. 


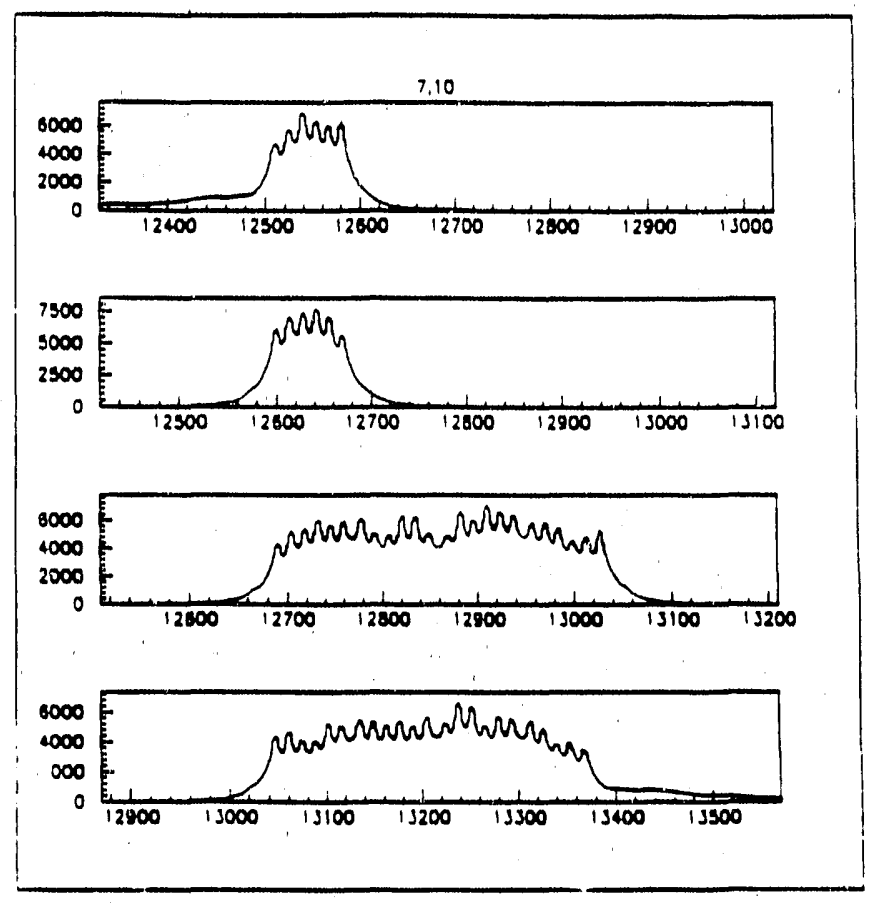

Figure 3-1: Kesults of radioactive source scan on one of the towers of the participant calorimeter. The current in a PM tube is digitized (shown here as the ordinate) as the source is drawn past the scintillator tiles. The abscissa is the position of the source, as measured in an optical encoder driven by the source transport system. The peaks correspond to individual scintillator tiles. The top two plots are for the EM sections, and the lower two plots are for the hadionic sections.

corresponding to the 59 scintillator plates, 6 in each of the two EM sections and 24 and 23 in the two hadronic sections. The amplitude of the peaks is proportional to the strength of the optical coupling. Fox has carried out a fitting procedure (see Figure $3-2$ ) to determine these constants for the EM sections. Introducing these amplitudes into the EGS Monte Carlo program. it has been possible to reproduce very well the electron response of the calorimeter for the yarious towers (see Figure 3-3. where the fraction of energy seen in EM1 is sinown for the different size towers at two different energits). Until this program was carried out. the lower-to-lower variation was not understood.

By scanning the radioactive source through the entire calorimeter, it was found that in the EM section. Out of 256 towers. 127 had all of their 6 plates coupled well. while 8 towers had lost all of the coupling: in the hadronic section. which has 24 plates for each tower. only 70 


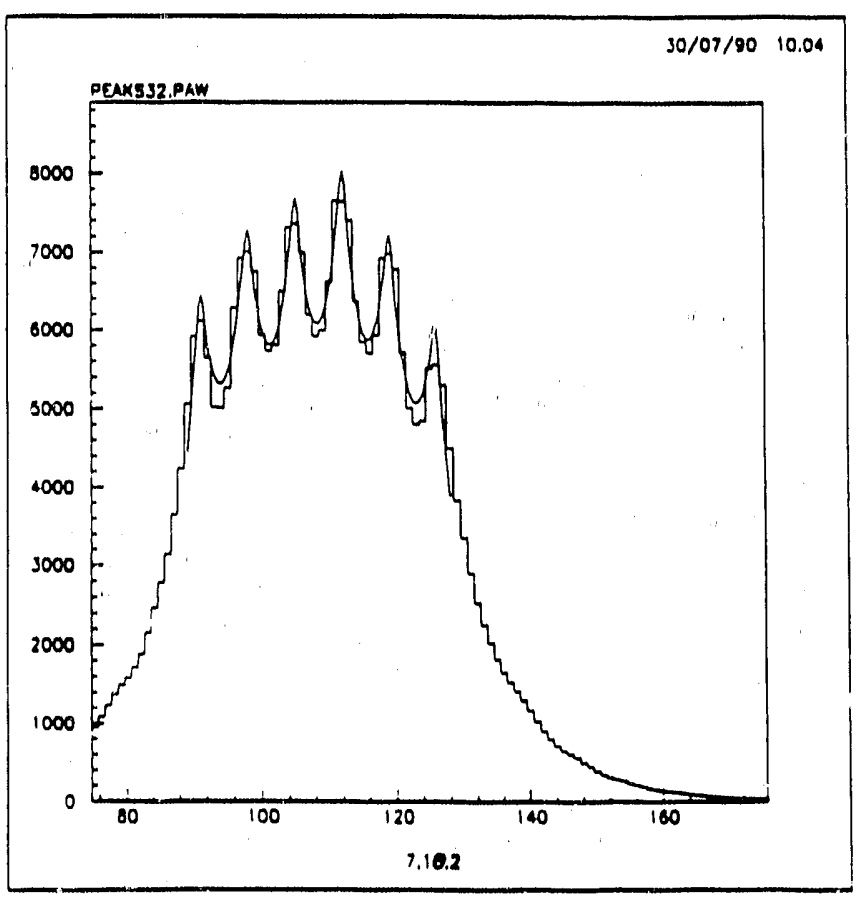

Figure 3-2: Data from the EM2 section of the tower shown in Figure 3-1 in which a fit for the optical coupling constants has been made. An exponential falloff has been included to account for contribution of the source to the current when it is beyond the actual scintillator plate. The amplitudes of the peaks are proportional to the optical coupling strengths. and these values are fed into the EGS Monte varlo to simulate the response $i$, the calorimeter.

out of 256 towers had perfect coupling, ? had only 14 plates coupled. the others were in between. It is evident from this experience that a scintilizior calorimeter in the which the light is carried to the photomultiplicr tubes via optical fibers must have a method to monitor the optical coupling in an automated way, as the system is very sensitive to optical coupling failures.

The radioactive source system appears to work very well, and the information it provides is uscrul in predicting the calorimeter response. However, it cannot be used frequently to detect PM gain shifts, since it takes approximately 1 hour to move the source through each quadrant of the calorimeter. We therefore have installed a DC optical source, which. although it cannot provide information on the light coupling between the scintillator and phototube does provide information on photomultiplier gain shifts. The optical ssurce mentioned above was designed 


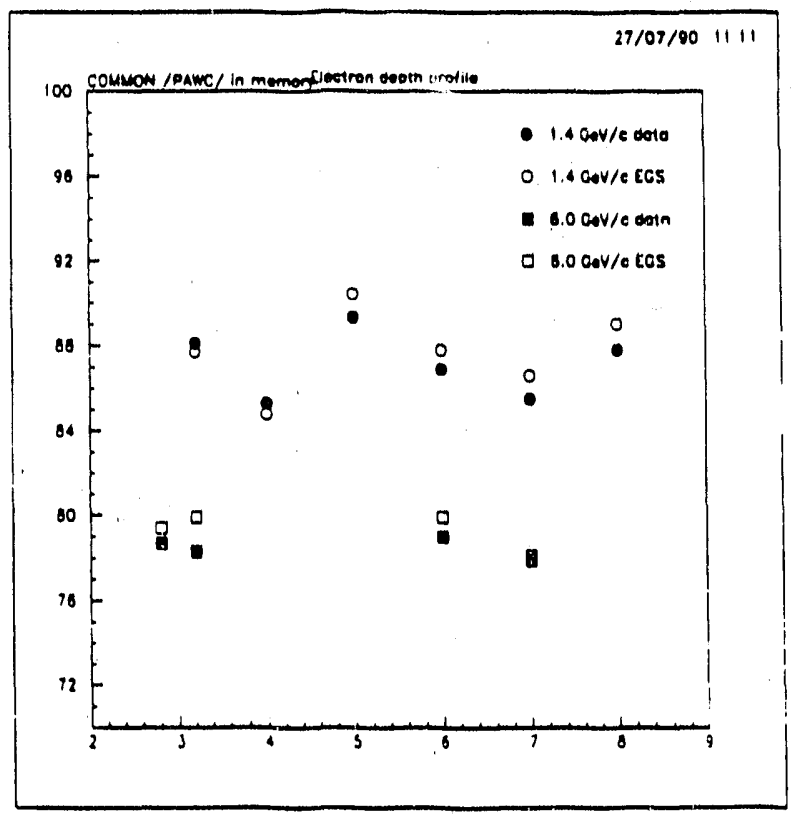

Figure 3-3: Fraction of the energy seen in. EM2 for seven of the calibration towers at two different electron beam energies. The Monte Carlo calculations include the optical constant obtain from the fits of the type shown in Figure 3-2. The tower-to-tower variations are clearly explained by the differences in optical coupling constants.

and constructed at Pitt by $D$. Kraus. The basic principle is to excite a WLS fiber of the same type as that used in the calorimeter (thus giving the same spectrum as light from the scintillator) with a DC lamp, and to fan this signal out to a large number of photomultiplier lubes as well as a PIN diode used for monitoring. In the source distribution box. the light is split nine ways--eight low level signals are routed to the light distribution ports already present in the PCAL. each of which distributes light to 16 PM tubes. and the remaining fraction of light (about 90\%) is sent to the PIN diode. The current from the latter is measured without amplification in a current monitor of the same type used by the PM tuoes. Thus the stability of the monitor is limited only the the characteristics of the PIN diode and the current monitors, both of which are excellent. In his report at the collaboration meeting. D. Fox also compared ine gain shifts measured with the cobalt source with those measured using the optical source. He found that there is in general very good agreement. as long as the current measured 
in the PMT is at least as large as the current measured in the PIN diode. A suinmary of his results, in the cases where sufficient current from the optical source is seen, is shown in a able 3-1. We conclude that the two systems track each other with an accuracy which exceeds the best energy resolution we can expect from the device (2\%).

Table 3-1: Comparison of the gain shif ts measured using the radioactive source with those measured by the optical source over three different periods of the June 1990 run.

$\begin{array}{lcc}\text { Length of period } & \text { Cobalt source } & \text { Optical source } \\ 3 \text { days } & 1.01 \pm 0.32 \% & 1.28 \pm 0.35 \% \\ 10.5 \text { days } & -0.82 \pm 0.34 \% & -0.12 \pm 0.61 \% \\ & & \\ & & \\ 11.5 \text { days } & -0.72 \pm 0.35 \% & -0.78 \pm 0.57 \%\end{array}$

In order to self-trigger the participant calorimeter on transverse energy, signals from each tower are weighted according to the mean angular position of the tower, as seen from the target. A sum of such weighted signals crcates an analog signal whose amplitude is proportional $\mathrm{i} E_{\mathrm{T}}$ seen by the calorimeter. A set of such summing circuits have been built and installed on the calorimeter, but so far, no good tests of the weights has been made. Therefore. a test sysiem was built to check the weight of every channel in the summing boxes. 4 pulse simulating the photomultiplier output was generated with a mercury relay driven by a DAC. The pulse was injected into each channel of the summing boxes and measured by the FERAs used for the trigger system. It was found that the existing weights were approximately correct but well outside the expected resolution of the calorimeter. A histogram of target weights minus measured weights is shown in Figure 3-4. The distribution is centered near unity 
but has a width of about 20\%. Before the next run, in which the geometry of the participant calorimeter will be changed to accommodate a larger aperture for the forward spectrometer, new weighting resistors will be installed, and the weights will be verified with the new test system.

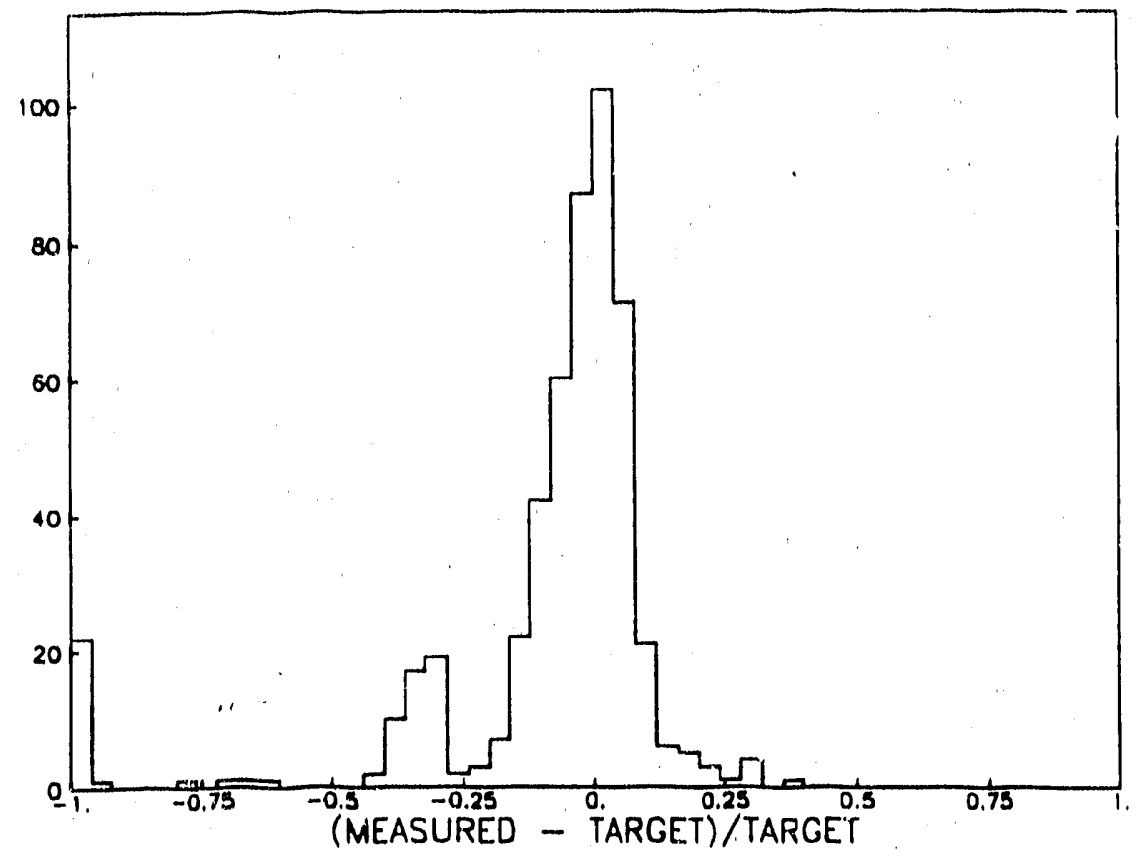

Figure 3-4: Histogram of the difference between the measured weights and the target weights in the summing circuits for the participant calorimeter.

A study of the PCAL $E_{\text {r }}$ spectra taken in the June 1990 run is under way. Uncorrected $E_{\text {T }}$ distributions made with the on-line calibration constants are shown in Figure 3-13 and also in Figure 1 of Appendix $\mathrm{C}$.

\subsection{Multiplicity Counter}

A feature of the original E814 proposal was the inability to measure charged multiplicity, as the tracking chambers were placed in the forward region. with small solid angle coverage. When it became clear that the participant calorimeter construction would be seriously delayed. the collaboration decided to add a modest multiplicity detector covering the rapidity region $1<\eta<4$ to provide a central trigger. However, in recent years. there has been a new interest in 


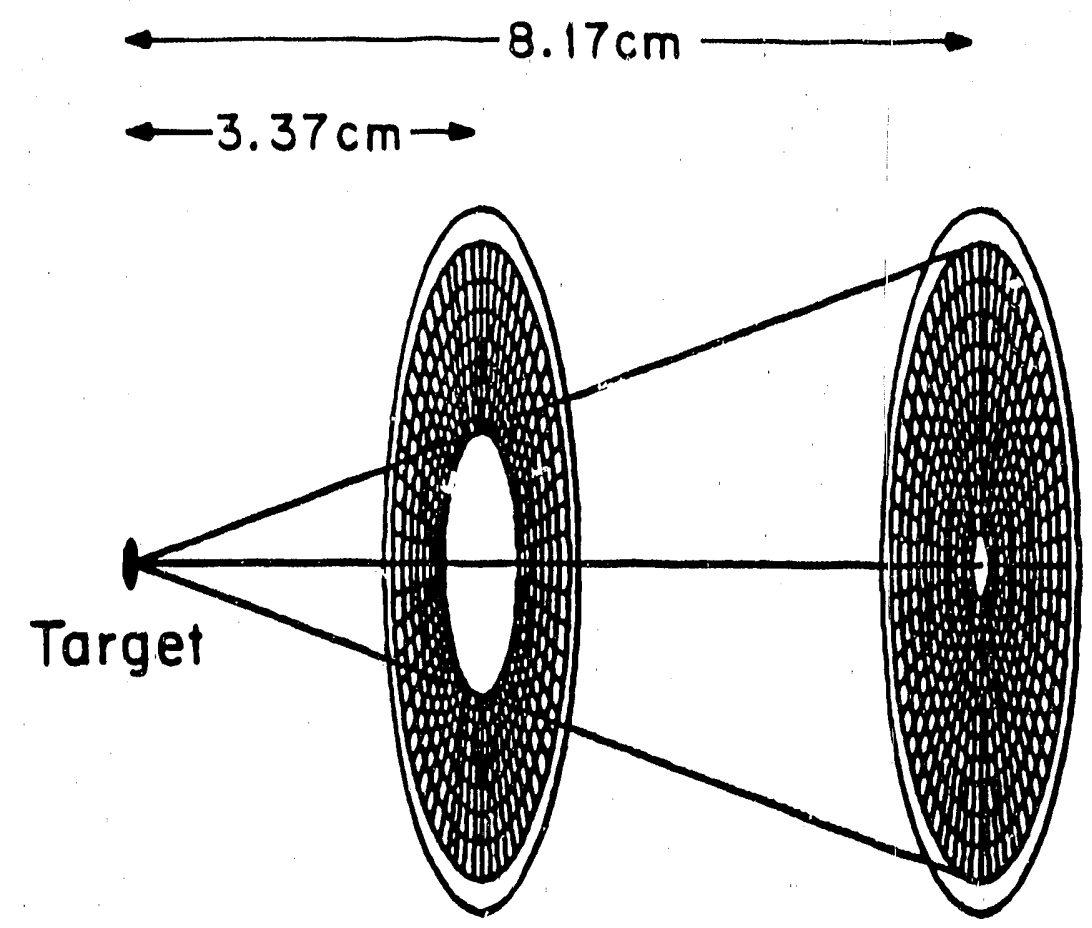

Detector $1 \quad$ Detector 2

Figure 3-5: An axonometric drawing of the two detectors

the pseudorapidity distributions themselves and the rapidity interval dependence of multiplicity distributions. Some experiments (E802 [11], E810 [12], HELIOS [13] and WA80 [14]) have published experimental results, and theoretical publications on the subject are numerous. The charged particle multiplicity distributions in general are believed to fit well negative binomial distributions. Some models have been suggested that can predict the nature of the collisions from these negative binomial parameters. Another important quantity that gives information on the nature of the collision is $E_{T} / n_{\text {ul }}$ data. It is known that in general $E_{\text {, }}$ and charged multiplicity track each other to a high degree of accuracy although the nature of events which fluctuate from average ratio has not been studied. 
The E814 multiplicity detector consists of two silicon pad detectors each with 512 pads. The segmentation of the detector was designed to approximately match that of the Participant Calorimeter, so that the information on $E_{\mathrm{r}}$ and multiplicity in the same region of solid angle is available at the trigger level. Also the radial segmentation of this detector is ideal for study of limited rapidity interval dependence of multiplicity.

The design of the geometry for the detector was based upon the principle of obtaining approximately equal counting rates in each of the pads while matching the azimuthal boundaries with the sectors of the trigger sums of the Participant Calorimeter. Preliminary multiplicity data from Experiment 802 was used for this purpose. The detectors are approximately $7.5 \mathrm{~cm}$ in diameter and 300 microns thick. Figure 3-5 shows the orientation of the detector with respect of the target.

The information read out from these detectors was in the form of a single bit, which was set depending on there was a hit or not on each pad. Fast analog sums of the total multiplicity and multiplicity on each sector (16 sums from each detector) were used by the trigger processor for triggering on central events. The data currently being analyzed were taken in June 1989 and June 1990. Central collision data were taken with six parallel physics triggers, three with different cuts on multiplicity and three with different cuts on $E_{\mathrm{T}}$ from the Target Calorimeter. The beam was $14.5 \mathrm{Gev} / \mathrm{N} \mathrm{Si}$ and the targets were $\mathrm{Al}, \mathrm{Cu}$ and $\mathrm{Pb}$.

Figure 3-6 shows the $d_{\sigma} / d n$ spectra for $\mathrm{Al}, \mathrm{Cu}$ and $\mathrm{Pb}$ targets as measured in the June 1989 run. These data have been corrected for the effects of dead pads, noisy pads and multiple hits. The effect of the dead pads and multiple occupancy is that the experimental multiplicity distribution for a set of events with fixed multiplicity is nearly Gaussian with a mean less than the input multiplicity. The correction to this was done by rescaling (event by event) the 


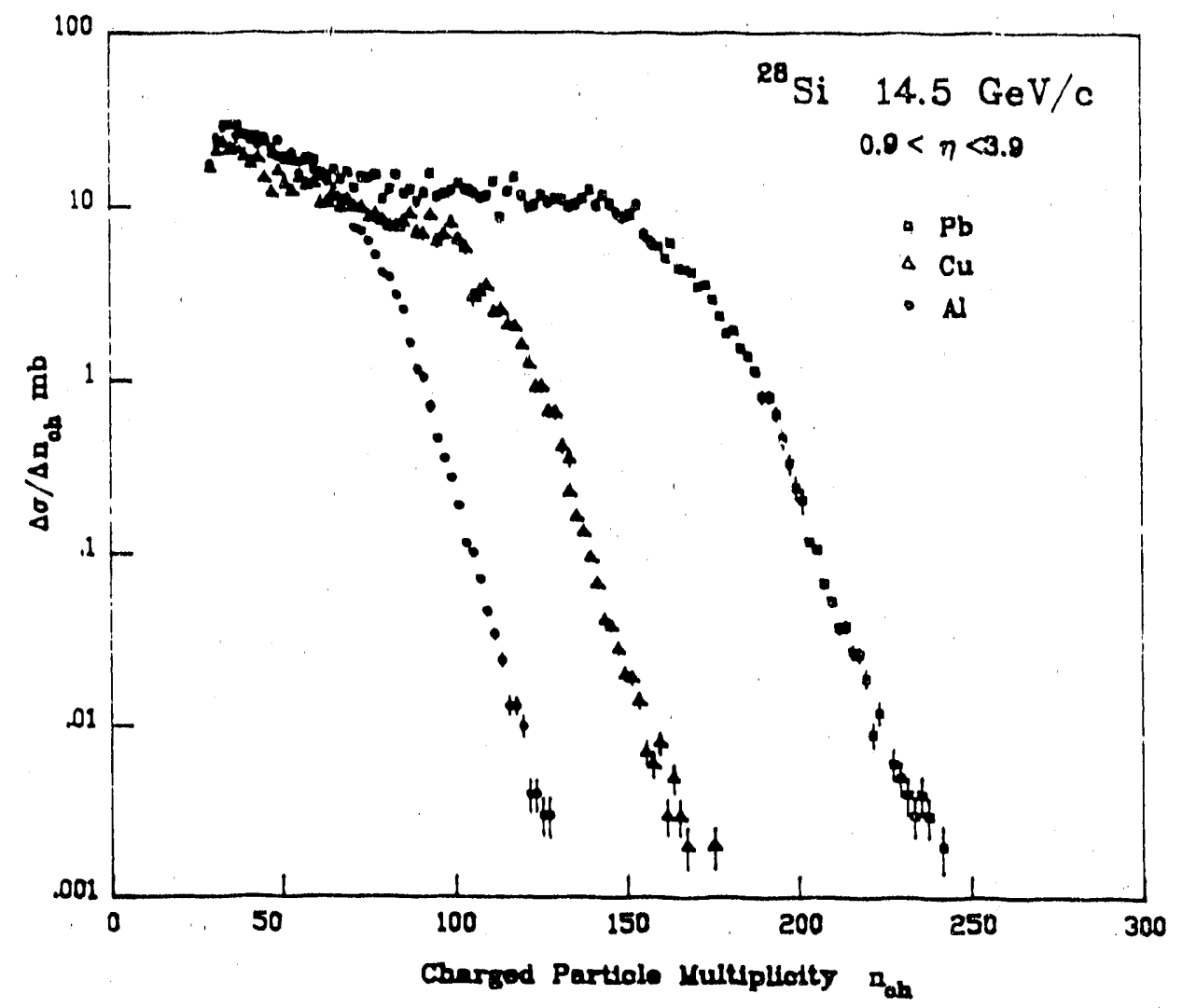

Figure 3-6: Measured $d \sigma / d n$ for $\mathrm{Si}$ beam with $\mathrm{Pb}, \mathrm{Cu}$ and $\mathrm{Al}$ targets from June 1989 run

number of hits on each ring for the number of dead pads and the mean multiple occupancy in that ring.

A comparison was done with LUND model FRITIOF (a version using the parameters modified by Gyulassy and later by the E802 collaboration [15]). While the plateau region is in agreement with data. the high multiplicity tail is badly underestimated by FRITIOF. A major reason for this is the absence of rescattering of secondaries and struck nucleons. which increases the multiplicity in the backward region.

The correction to dead pads and multiple occupancy described above was lested using 


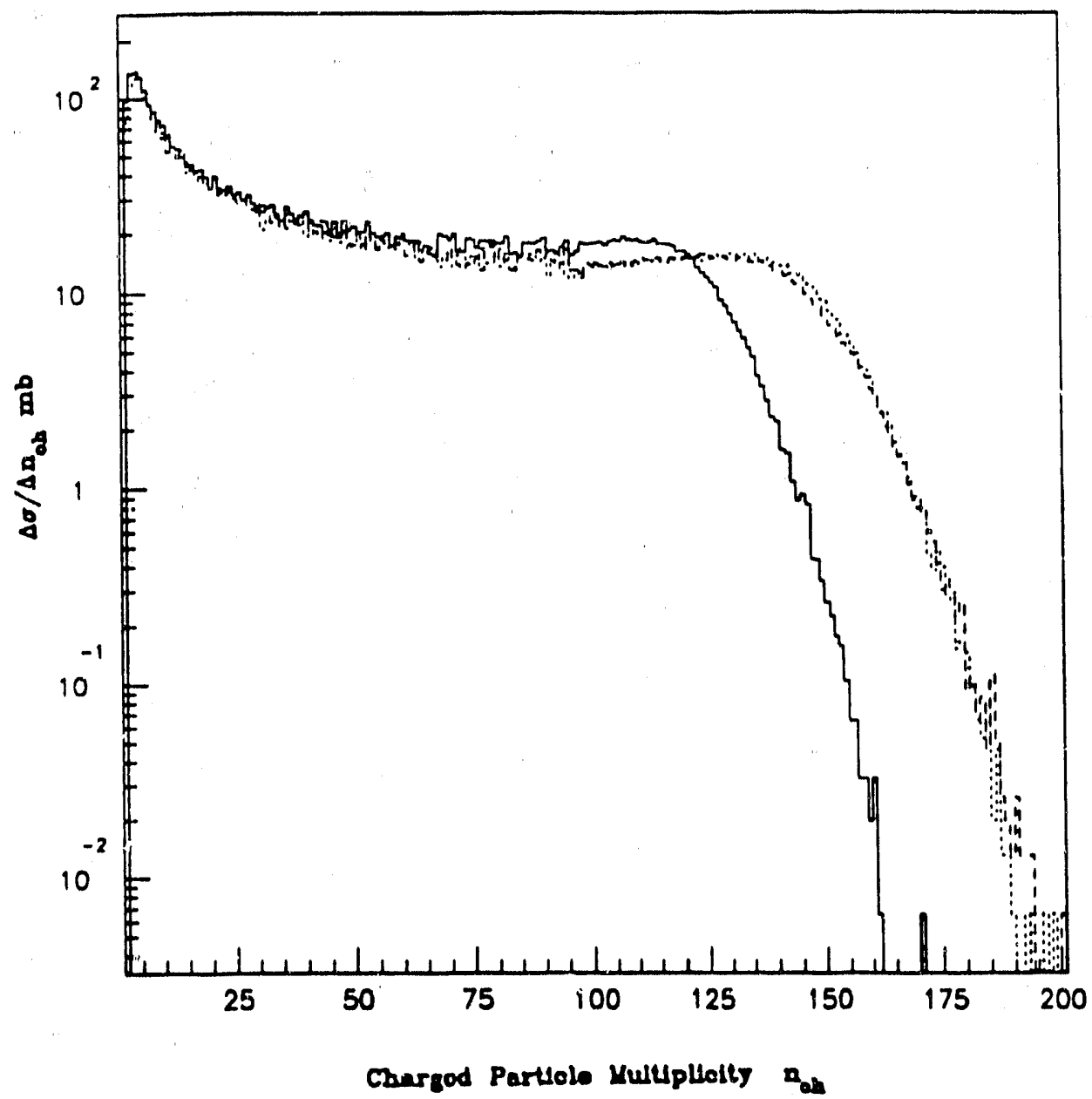

Figure 3-7: Test of dead pad and multiple occupancy correction to $d \sigma / d n$ curve using FRITIOF ( $\mathrm{Si}$ beam on $\mathrm{Pb}$ target)

FRITIOF. First $d \sigma / d n$ was calculated with dead pads included in the description of geometry of the detector and the resulting events were corrected using the same procedure used for real data. The do/dn curves with no dead pads. with dead pads but before correction and with dead pads after correction ( $\mathrm{Pb}$ target) are shown in Figure 3-7. The true spectrum and the corrected spectrum agree very well.

Figure 3-8 shows the $d n / d \eta$ is. $\eta$ plots for the targets $\mathrm{Pb}$. $\mathrm{Cu}$ and $\mathrm{Al}$ with three different multiplicity cuts from June 1989 run. A striking feature of these plots is the discontinuity of the curves at approximately 1.8 pseudorapidity where the interface of the two detestors is located. Although it was found that many types of events could produce this effect (upstream 

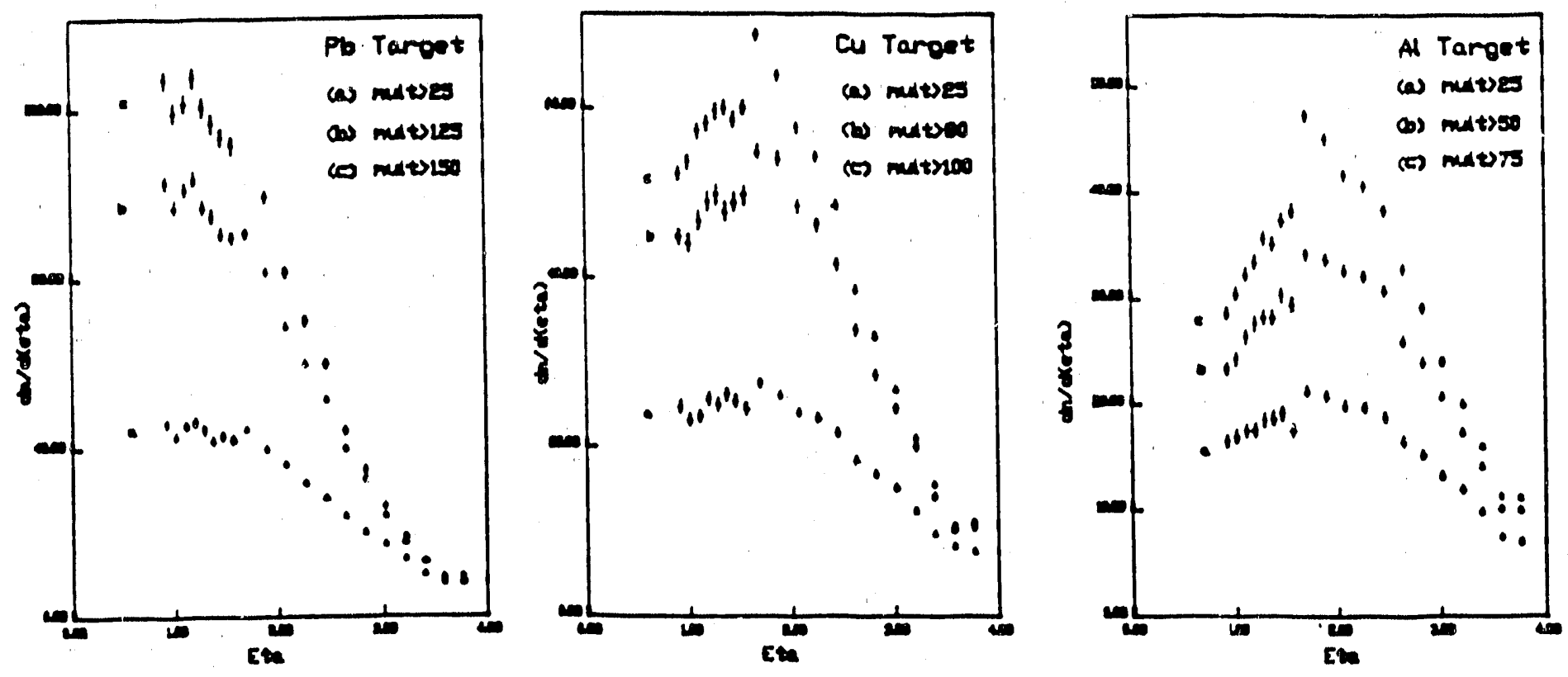

Figure 3-8: $d n / d \eta$ plots for $\mathrm{Si}$ beam on (a) $\mathrm{Al}$ (b) $\mathrm{Cu}$ and (c) $\mathrm{Pb}$ targets for three different multiplicity triggers (June 1989)

interactions, beam particles hitting the first multiplicity detector. and certain types of noisy events which could be eliminated by recognition of their characteristic patterns) it was not possible to eliminate it totally in the offline analysis. The major reason for the higher $d n / d \eta$ at the second detector was suspected to be secondary interactions of heavy fragments at the non-active central region of silicon in the first detector. In the June 1990 run this non active region of silicon was removed and the resulting $d n / d \eta$ curves, which do not have the discontinully, is shown in Figure 3-9.

An analysis of negative binomial properties of multiplicity distributions was done using a method suggested by Giovannini and Van Hove [16]. Negative binomial distributions are characterized by

$$
P(n,<n>, k)=\frac{k(k+1) \ldots(k+n-1)<n>n k^{h}}{n !(<n>+k)^{n+k}}
$$



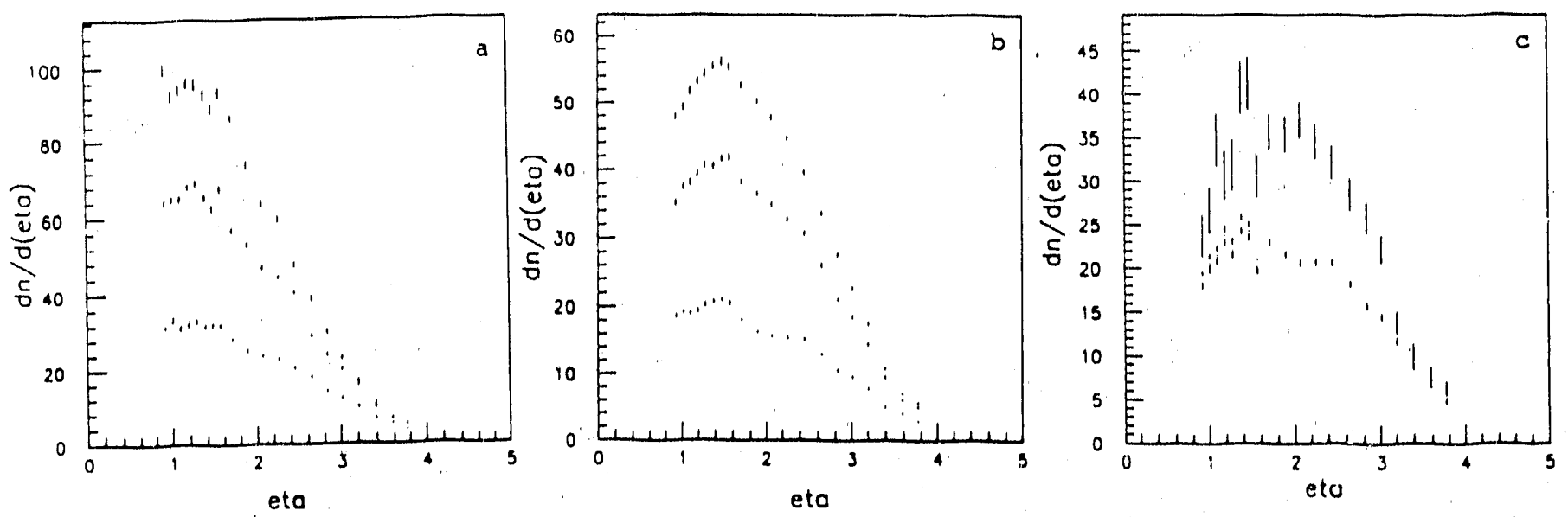

Figure 3-9: $d n / d \eta$ plots for Si beam on (a) $\mathrm{Al}$ (b) $\mathrm{Cu}$ and (c) $\mathrm{Pb}$ targets for three different multiplicity triggers (June 1990)

in which $\langle n\rangle$ is the average multiplicity, and $k$ is related to $D^{2}=\left\langle\pi^{2}\right\rangle-\langle n\rangle^{2}$ by:

$$
\frac{D^{2}}{\langle n\rangle:}=\frac{1}{\langle n\rangle}+\frac{1}{k}
$$

In Giovannini and Van Hove [16] it is suggested that a recurrence relation between $F(n)$ and $F(n+1)$. where $F(n)$ is the frequency of occurrence of multiplicity $n$ in a large number of collisions. can be written as

$$
g(n)+\frac{(n+1) F(n+1)}{F(n)}
$$

The quantity $g(n)$ can be shown to be a constant a for Poisson distribution while it becomes $a+b n$ for negative binomial distribution. with $a=<n>k /(<n>+k)$ and $b=\langle n>i(\langle n>+k)$.

Using E814 multiplicity distributions. $k$ and $\langle n>$ were calculated for different rapidity 
intervals. This was done by fitting a Gaussian to multiplicity distribution in each rapidity interval and then calculating the nefative binomial parameters from $D$ and $\langle n\rangle$ using the relation shown above. Plots of these parameters are shown in figure 3-10.
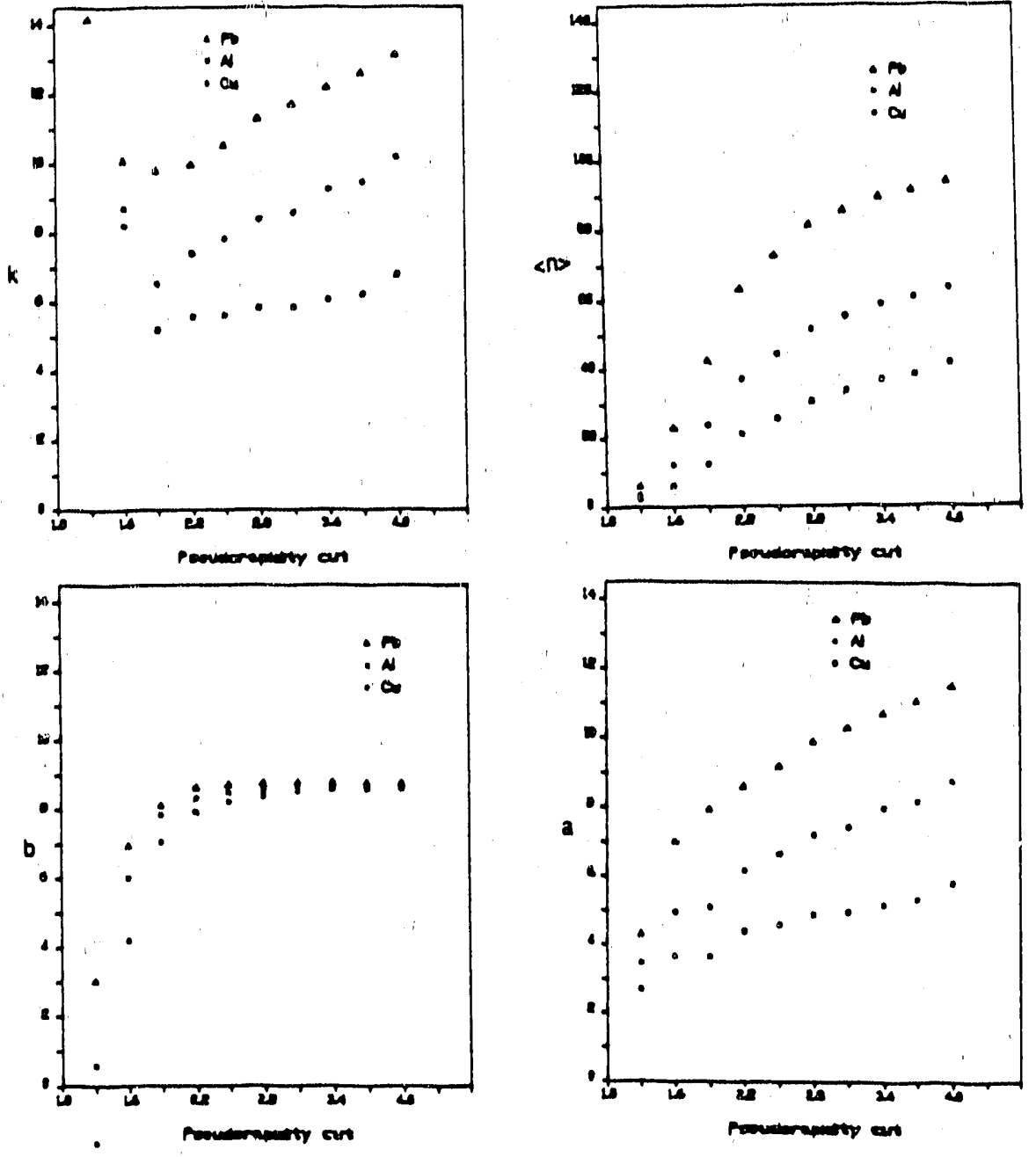

Figure 3-10: Parameters $a, b . k$ and $\langle n\rangle$ calculated from E814 multiplicity distributions in different rapidity intervals. All intervals start from rapidity 1 and are not symmetric.

Two different models are discussed in Giovannini and Van Hove [16] to interpret the behavior of the parameters $a, b . k$ and $\langle n\rangle$. According to the first model, partial stimulated emission of bosons. if $n$ particles are present at a given stage of a collision. emission of the $(n+1)^{\prime \prime \prime}$ particle can be independent of those already present or it can be emitted due to BoseEinstein interference. The term $a$ is due to the first of these effect while the latter effect 
gives rise to the term $b n$ in $g(n)$. In this model. $1 / k$ is the fraction of particles already present stimulating the emission of an additional particle. When the rapidity interval gets larger. since the range of Bose-Einstein interference is finite, the fraction $1 / k$ decreases as seen in the plot of $k$ vs rapidity interval.

In the second (cascade) model, a certain number of particles (ancestors) are assumed to be originally produced. Then more particles are emitted by them. where the former particles can change their momentum and quantum numbers. A cluster is formed of all particles originating directly or indirectly from one particle regarded as being originally produced. The particles observed in a given rapidity region may be produced by the original particles that were in the same region or by ancestors that were outside ihs region. The former effect causes the term $b n$ in $g(n)$. which is proportional to total multiplicity while the constant term is due to the latter effect. When the rapidity interval increases for a given multiplicity $n$, the probability that a given particle is produced by an ancestor inside the same region increases. Therefore. $k / n=a / b n$ increases with the size of the rapidity interval.

The parameter $a$ in the cascade model is proportional to the number of ancestors and parameter $b$ describes the distribution in one cluster originating from a given ancestor. When the size of the rapidity interval is increased. the fraction of each cluster included in the observed region grows increasing $b$ up to a limit. When the observed interval is larger than a full cluster $b$ becomes constant. However the number of clusters included in the rapidity interval keeps increasing, as shown by the plot of $a$. The fact that $a$ shows a large target dependence in heavy ion collisions while $b$ has very little target dependence can be interpreted as the number of ancestors increasing when the larget mass is larger but all the ancestors cascade the same way independent of the target mass. Although these results are interesting, data used for this analysis had two drawbacks. First, the centrality of the events was 
determined by triggering on a combination of $E_{T}$ in the backward region and forward multiplicity which biases the distributions in an unknown way, as suggested by figure 3-11. Second. the step in the $d n / d \eta$ plots as shown in Figure 3-8 was present in this set of data. However, the results seem encouraging, and we plan to carry out a similar analysis with June 90. data which do not have either of the above problems.

Figlire 3-11 shows the multiplicity $(0.9<\eta<3.9)$ vs. $E_{T}$ measured in TCAL $(-0.5<\eta<0.9)$ vith the experiment triggered on $E_{T}$ (Figure 3-11a) and on total multiplicity (Figure 3-11b). The difference between the two distributions at high multiplicity or $E_{T}$ shows how the trigger can affect the shape of these distributions. When triggered on $E_{T}$ it selects events with high $E_{T}$ backward and less multiplicity in the forward region while the opposite happens when triggered on multiplicity.
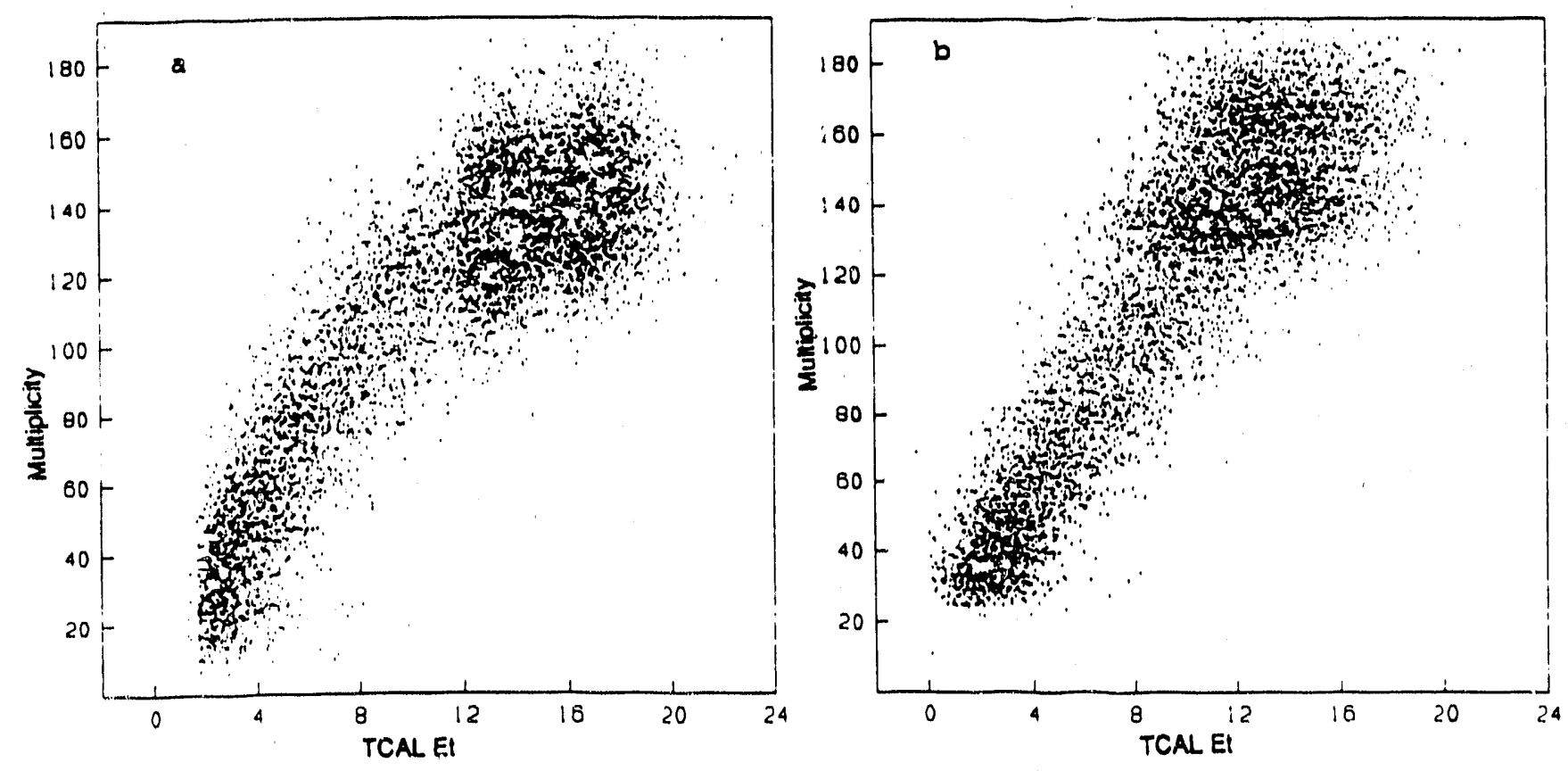

Figure 3-11: Multiplicity $(0.9<\pi<3.9)$ vs. $E_{T}$ measured in TCAL $(-0.5<\eta<0.8) \quad$ (a) triggered on $E_{T}$ and (b) triggered on multiplicity 
Figure 3-12 shows the total energy in a forward $0.8^{\circ}$ cone (as measured by UCAL) vs. multiplicity. The anti-correlation is expected, since when the projectile fully interacts with the larget giving rise to high multiplicity, the amount of energy going forward decreases. Figure 3-13 shows the correlation between the multiplicity and the uncorrected total $E_{T}$ from PCAL. which covers approximately the same rapidity region as the multiplicity detector. It is seen that $E$ is highly correlated with the multiplicity, as expected. At very high multiplicity region there is a non linearity in the correlation, which is not predicted by FRITIOF.

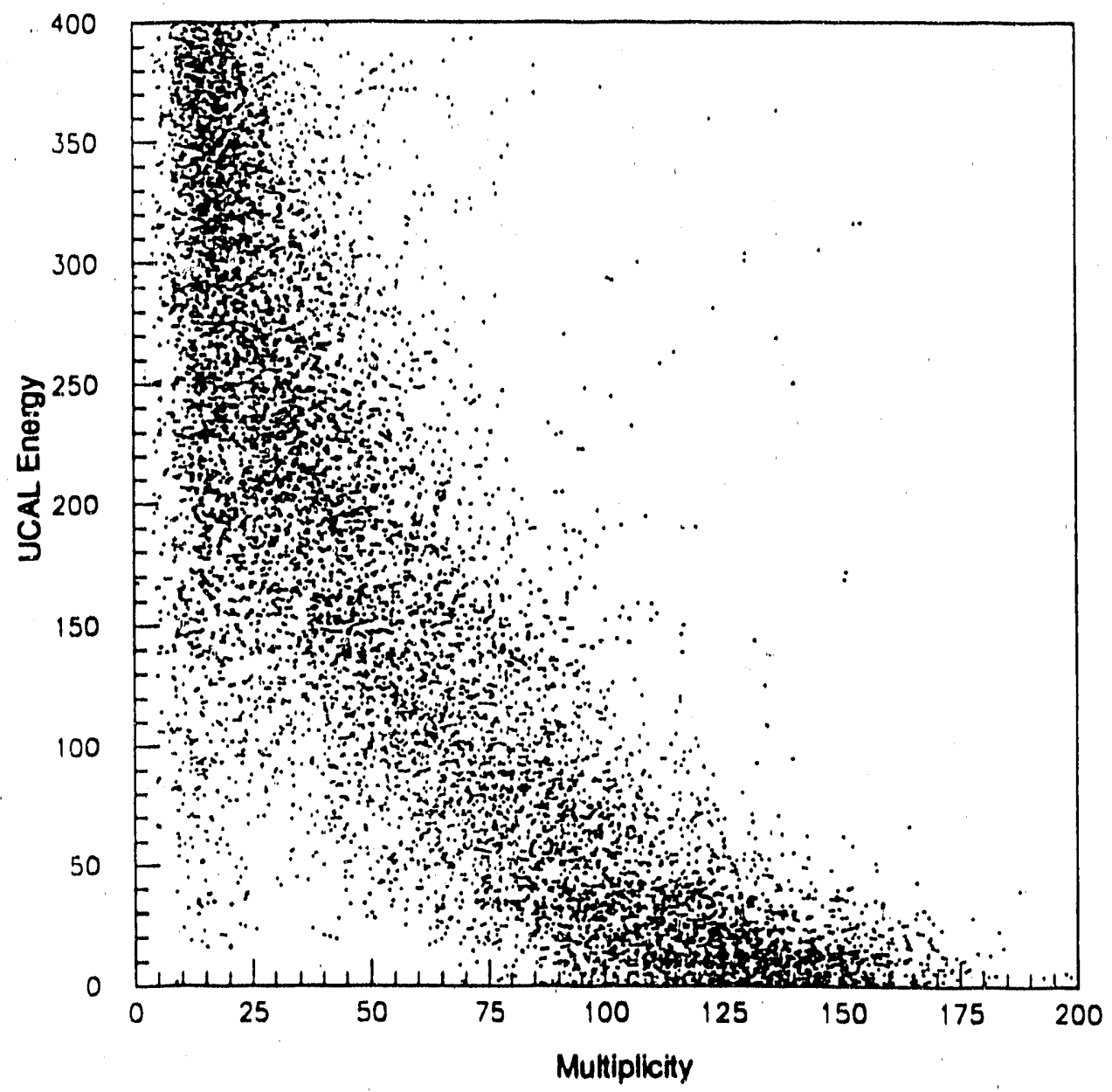

Figure 3-12: Total energy in a $0.8^{\circ}$ cone measured by UCAL is. multiplicity 

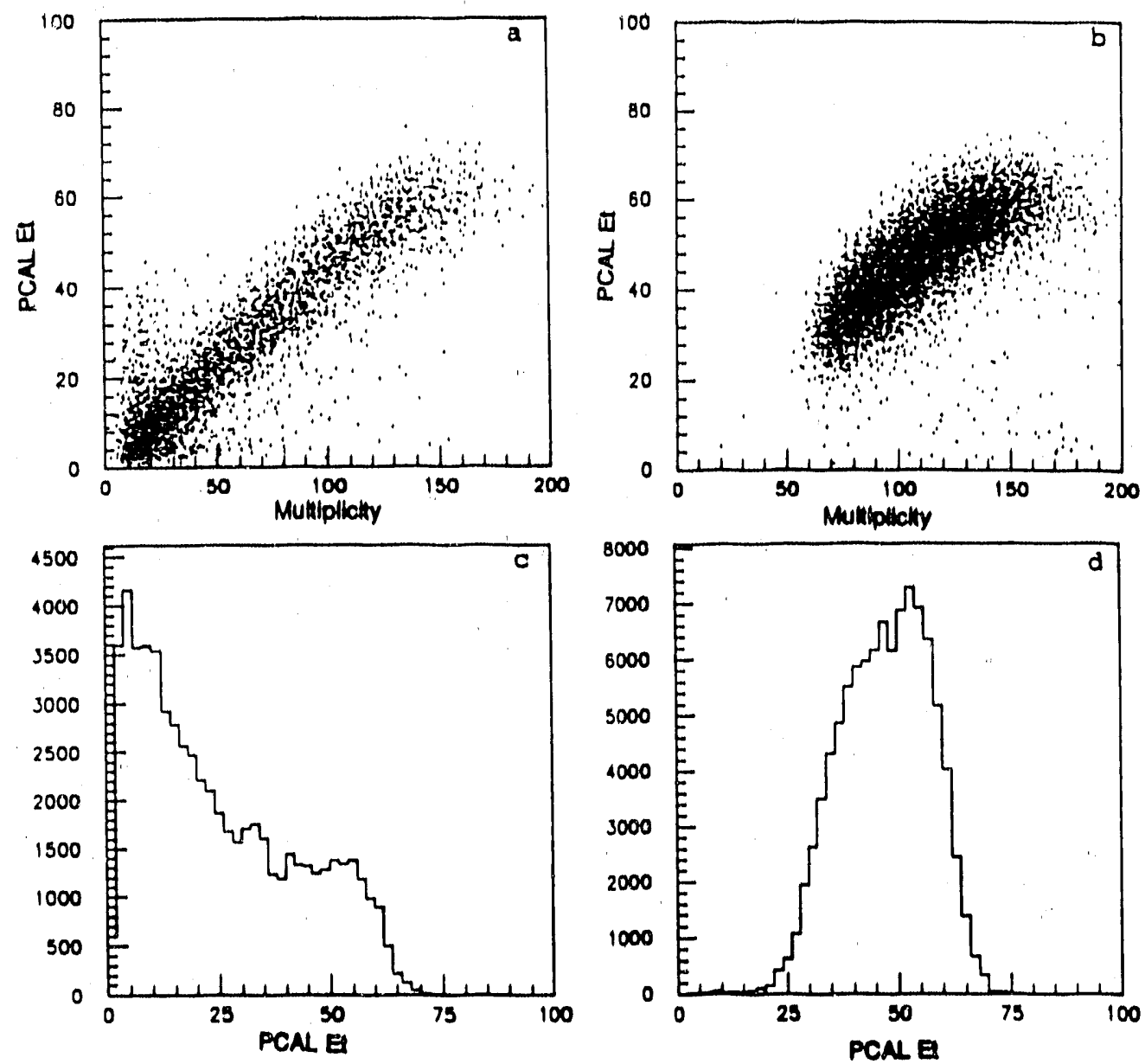

Figure 3-13: Total $E_{T}$ measured by PCAL vs. multiplicity for (a) multiplicity > 10. (b) multiplicity $>70$. (c) and (d) are the corresponding (uncorrected) PCAL $E_{\text {, }}$ spectra. The data were taken with a Pt target.

\subsection{Forward Scintillation Counters}

The University of Pittsburgh is responsible for the scintillation counters placed before the forward calorimeters (Forward Scintillators). These counters measure the charge of forwardgoing particles $(Z)$. the time of flight of these particles (TOF), and the vertical coordinate of the track (y). The University of Pittsburgh is also responsible for the magnet scintillation counters. which line the lateral walls of the spectrometer magnet channels. The magnet scintillators are intended principally to serve as veto counters in peripheral triggers but they are designed and instrumented so as to permit the measurement of charge as well as position along the beam line. from which a rough value for $p / Z$ can be inferred, and TOF. 


\subsubsection{Performance Criteria}

These Forward Scintillators must determine $Z$ to within one charge unit, measure the lime of f'light to better than $1 \mathrm{~ns}$, and find the track coordinates 10 within one calorimeter module $(10 \mathrm{~cm} \times 10 \mathrm{~cm})$. In conjunction with the charge-digitizing electronics they must accommodate a dynamic range of at least $300: 1$ above the discriminator threshold. Although performance may be improved by off-line correction (eg. slewing corrections for timing, and position-dependent corrections for pulse-height) they must provide the $\mathrm{Z}$ measurement in time for the generation of second-level triggers. The spatial resolution in $x$ is determined by the counter width, and in $y$ (or $z$ for the magnet scintillators) by demanding that a measurement of time difference between the counter ends have resolution of about 1 ns. Resolution in the measurement of $Z$ is determined by variation in the light yield as a function of position (principally a result of non-uniformity in thickness) and by statistical fluctuations in the deposited energy arising from the production of energetic delta-rays. The former effect can be corrected for off line. The attenuation length of NE104 $(120 \mathrm{~cm})$, which was selected for its good timing properties, and because it would permit an independent measurement of position by pulse height ratio, produces a $12 \%$ variation in the summed signal from the two ends of the counter. This factor in pulse-height variation can be removed by using the geometric-mean of the pulse heights from the two ends.

The variation in deposited energy has been analyzed by Badhwar [17, 18], with results implying that for heavily-ionizing particles, the dependence is:

$$
(\sigma / I)^{2}=\gamma s /\left(2 s Z^{h}\right)^{2}
$$

in which $s$ is the scintillator thickness, $k$ is determined by the scintillator properties. $Z$ is the incident particle charge, I the mean light yield, and $\gamma$ is given by:

$$
\gamma=\xi \varepsilon_{D}\left[2-\varepsilon_{D} / \varepsilon_{\max }\left\{\beta^{2} / 2+1+\beta^{2} \ln \left(\varepsilon_{\max } / \varepsilon_{D}\right)\right\}\right] .
$$

with 


$$
\xi=0.3(z / A) \quad\left(\mathrm{mc}^{2} / \beta^{2}\right) \mathrm{siz}^{2} \text {. }
$$

In these expressions $\varepsilon_{D}$ is the mean energy deposited by a delta ray that leaves the scintillator, and $\varepsilon_{\text {III, }}$ is the maximum delta ray energy. This expression approximates the effect of limited energy deposil because of finite thickness by an using an average range for delta rays leaving the rear surface, whatever their production point. For a $1 \mathrm{~cm}$ thick scintillator, we find that $\sigma / I \simeq 0.18 / z^{k-1}$, which yields a resolution of $2.6 \%$ at $z=16$, assuming that $k=1.7$, a value close to what we have observed in our tests of scintillation counters in a Si beam. For $Z$ less than $8 \mathrm{k}$ is near 1.9 ,

The conclusion of this analysis - that the variance is a factor of two to three below that implied by the Vavilov calculation of energy loss - has wide confirmation, although the details of the $Z$ dependence and the question of an appropriate choice for the $\varepsilon_{D}$ parameter are unsettled. The resolution implied by this expression has been observed in ionization chambers for $Z$ up to 50 [19], and at low $Z$ in scintillators [20]. It is consistent with the observations by E802, and with our observations.

\subsubsection{Electronics Chain}

Two signals are taken from each phototube. one from the anode and the other from the last dynode. The last dynode signals from the two ends of each counter are added and inverted. This signal is brought into the FERA, which is used in the trigger.

The anode signals from each PM are brought into the counting room and split passively between an ADC (LeCroy Model 1885) and a discriminator (Phillips Model 7106). The ECL outputs of the discriminators are fed to TDCs of $0.3 \mathrm{~ns} /$ channel resolution (LeCroy Model +291B). The timing measurements, which use constant threshold discriminators, are corrected for slewing offline using the measured pulse height. 


\subsection{3. $Z$ Identification for peripheral events}

In peripheral collisions identification of the final states of each decay channel provides a unique way to study reaction mechanisms which lead to different final states. In heavy ion cxperiment environments such as E814, this means, identifying simultaneously light products as well as heavy reaction fragments, giving rise to a large dynamic range in pulse height. In our case the spectrum spans the range from minimum lonizing particles $(Z=1)$, all the way to $Z=14$. This leads to severe requirements on levels of tolerated noise and pedestal stability.

After the initial reaction the charged light particles $(1 p, 2 p, 3 p$...etc) have a liight path of about $10 \mathrm{~m}$. while the heavy fragments go about $30 \mathrm{~m}$ before detection. Although the full flight path is covered with Helium gas in order to reduce intermediate interactions, about $40 \%$ of the heavy fragments interact, producing a variety of nucleons and nuclear fragments. For this reason, it is important to measure the charge of the reaction fragments immediately downstream of the target and to again verify the charge of the heavy fragment after the full flight path.

Figure 3-14 shows the detectors we used to identify reaction fragments in the June 1990 run. There were two Si detectors, one immediately upstream and the other immediately downstream of the target, in order to measure the charge of the incoming beam projectile and the outgoing reaction products. The reaction products are transported through two dipole magnets, which separate light and heavy reaction fragments. Light fragments are detected by the upstream scintillator hodoscope (10 vertical scintillator plates of size $10 \mathrm{~cm}$ by $60 \mathrm{~cm}$ ) and $5 \mathrm{Ur} / \mathrm{Cu}$ calorimeter stacks. Heavy fragments and neutral fragments ( 1 n.2n..etc) have a longer flight path and they are detected by the downstream scintillator hodoscope $(39$ lertical scintillator plates of $10 \mathrm{~cm}$ by $120 \mathrm{~cm}$ each) and $20 \mathrm{Ur} / \mathrm{Cu}$ calorimeter stacks. In addition to this, heavy fragments are detected by 5 horizontal scintillator plates of $10 \mathrm{~cm}$ by $120 \mathrm{~cm}$ each installed between the 
downstream scintillator hodoscope and the calorimeters as shown in the figure. These additional horizontal scintillator counters provide us with one more measurement of the charge of the heavy iragment as well as the $X$ position measurement with an position resolution of about $\sigma=2 \mathrm{~cm}$. corresponding to about a 0.5 milliradian angular resolution.

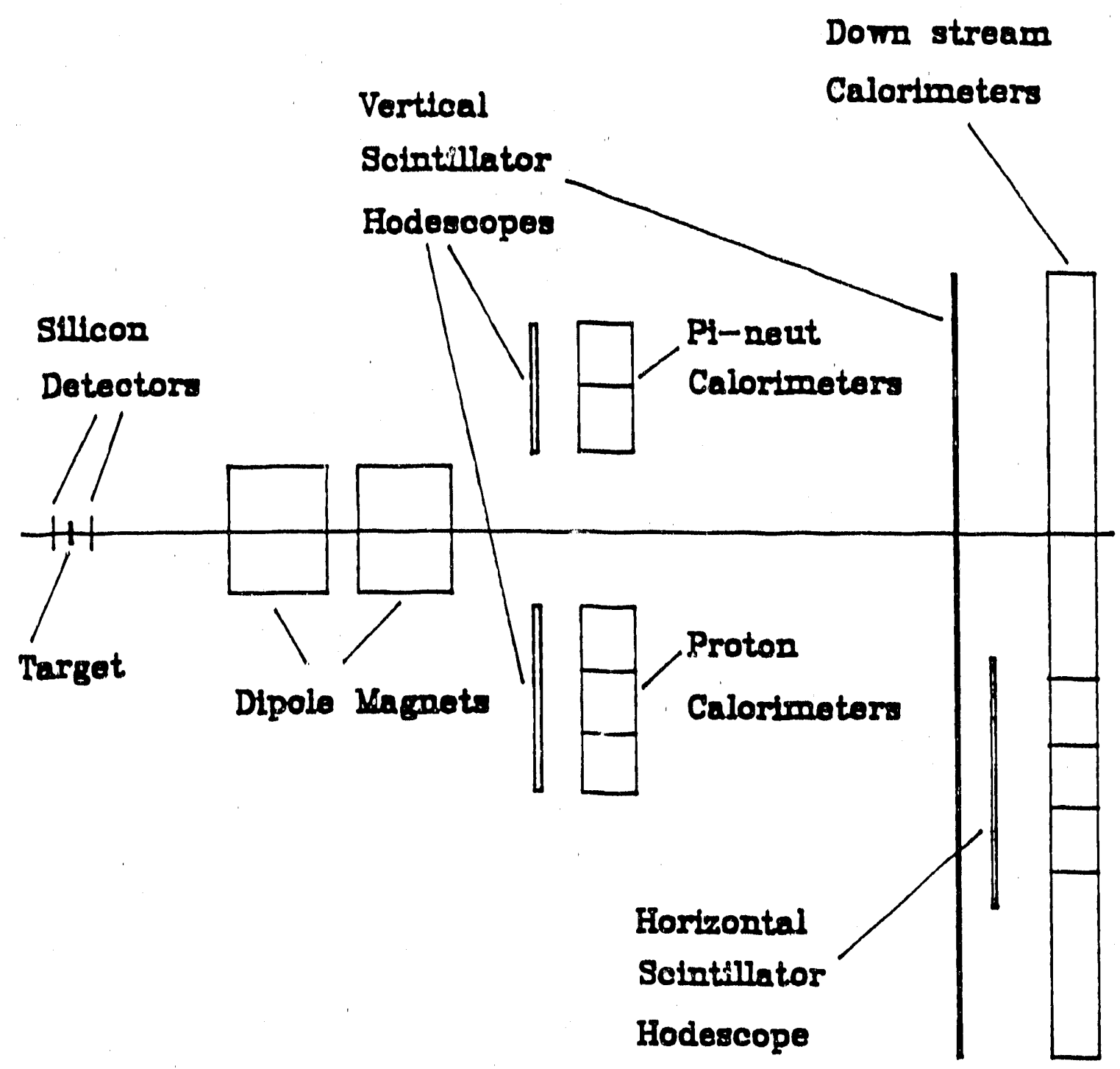

Figure 3-14: Schematic of the E814 detector layout during June 1990 peripheral run which were used for the charge measurements 
Figure 3-15(a) shows a measured charge distribution for the sillicon detector downstream of the target, plotted against the charge measured tin the counter in thit downstream hodoscope through which the beam passes (counter v20). Figure $3-15(b)$ shows the same distribution for the counter in the horizontal scintillator hodoscope through which the beam passes (counter H3). In both counters the minimum ionizing peak was calibrated to be at channel 100 . Each island of points corresponds to a charge fragment. Since the pulse height in the silicon detector varies as $Z^{2}$, the slight curvature in these plots indicates a departure from $Z^{2}$ behavior for the scintillation counters, attributable to saturation effects in the scintillator material. This effect is very strong in the counter $\mathrm{H} 3$.
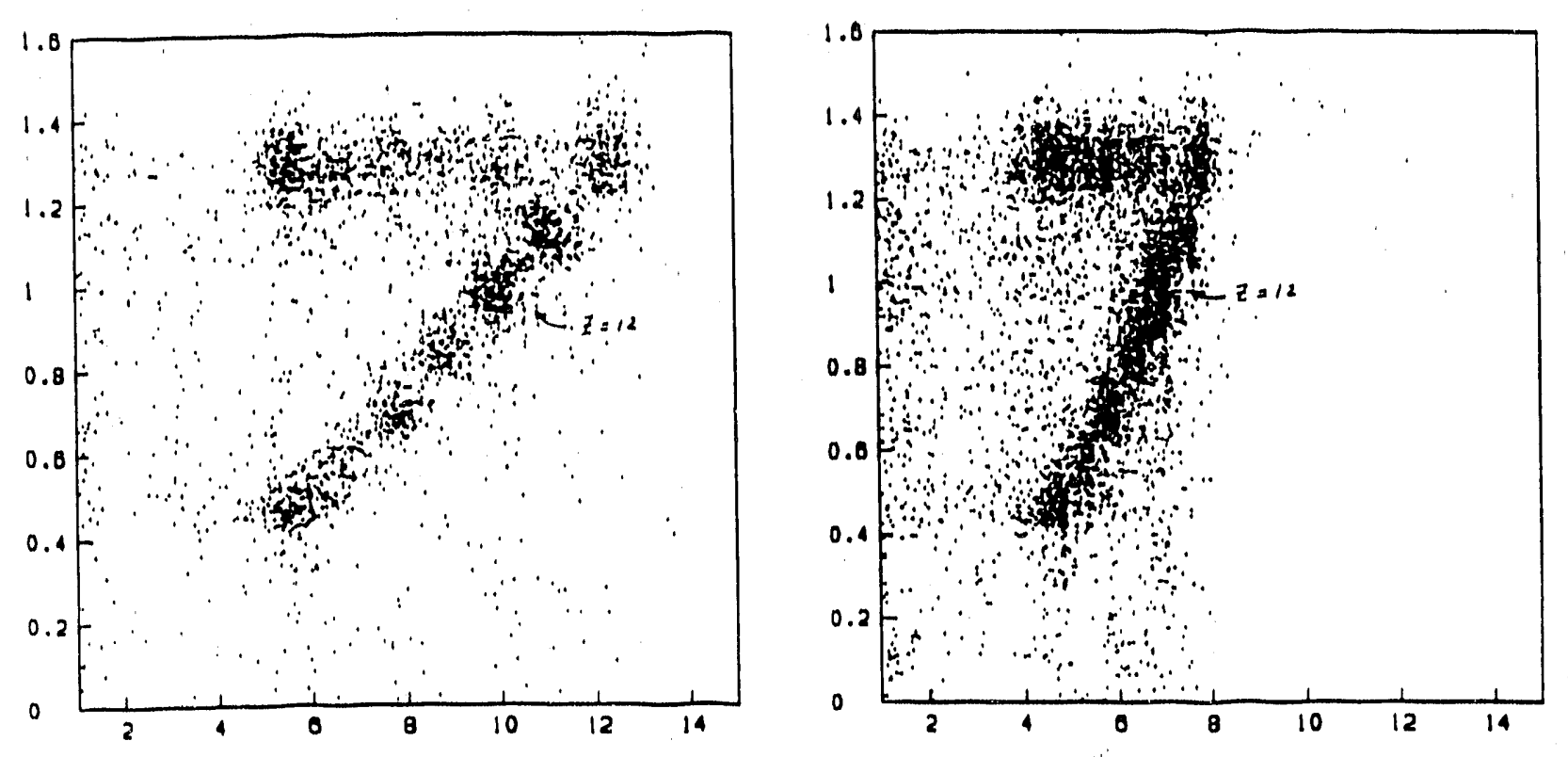

Figure 3-15: (a) Measured charge distribution for Si detector vs vertical counter V20. (b) Same distribution with horizontal counter H3. Beam passes through both counters.

These plots demonstrate very clearly that the events which leave as $Z=14$ after the target. interact downstream and change their final state. These are the events which form a horizontal band of islands in the plots. Only the events which are correlated in both detectors are good candidates for events interacting in the target. Events which have $Z<8$ were rejected in the trigger in searching rare channels. 
Identifying heavy fragments which survive all the way the downstream. can be done by selecting the correlated events in the silicon detector just downstream of the target and the scintillator hodoscopes. As described above. the relationship of $Z$ to pulse height in each counter is different due to saturation effects. These effects mainly comes from phototube saturation and scintillator material saturation. One solution for this effect is to remove saturation effects using Birks parameterization [21] which was developed for heavily ionizing particles at low velocities given by

$$
(d E / d X)=\frac{A(d E / d x)}{(1+B d E / d x)}
$$

Here $(d E / d X)_{\text {ill, }}$ is proportional to the quantity of light produced for a particle of given ionization $d E / d x . \quad A$ and $B$ are saturation parameters which depend on the scintillator material.

We used this parameterization to map each counter to one common scale (in this case the downstream silicon letector) by evaluating the constants $A$ and $B$ for each counter separately. Figure 3-16(a) shows a plot between vertical and horizontal hodoscopes after this correction for the $\mathrm{Z}$ measurement was made. Good candidates lie along a $45^{\circ}$ line indicating a strong correlation between each measurement. Events which lie on $X=0$ or $Y=0$ corresponds to the hits where one of the measurements fails to report and the other succeeds. Figure 3-16(b) shows the $Z$ spectrum after this doliole charge measurement. A.ll charges from 8 through 14 are clearly visible in the plot.

Figure $3-17$ (a) shows the plot between the silicon detector just after the target vs the double Z measurement after Birks corrections. All events which lie on the $45^{\circ}$ line are gocd candidates. Events which lie as a horizontal band are downstream interaction products. Figure 

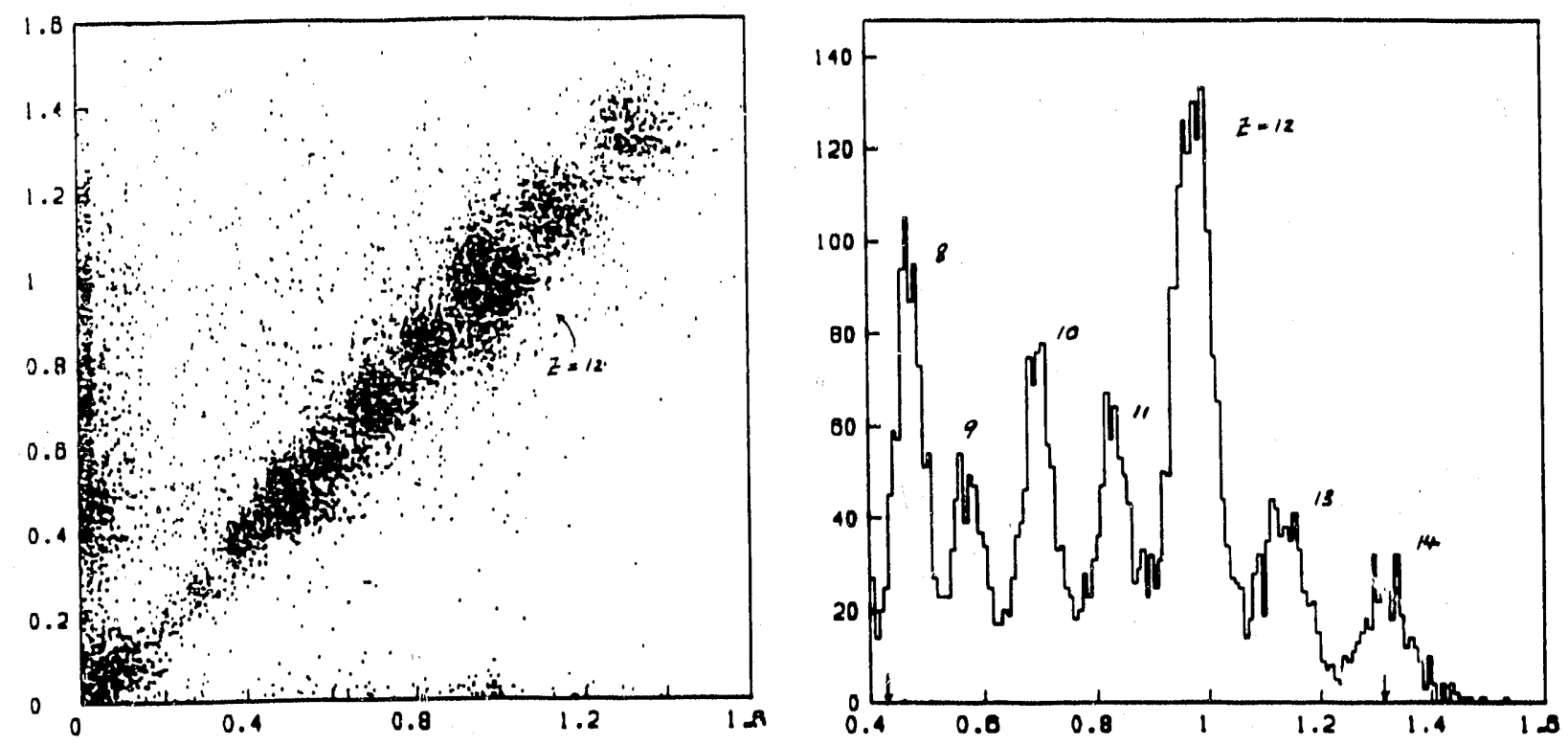

Figure 3-16: (a) Correlation between vertical and horizontal scintillator hodoscopes after Birks parameterization (b) $Z$ spectrum after combined measurement (double charge measurement)

$3-17(b)$ shows the triple charge spectrum obtained by combining these three Jetectors. The peak to valley ratio is much better in this plot indicating a clear separation of charges. This is clear from the downstream interaction and represent very good candidates. The resolution of the triple charge measurement is about $2.5 \%$ for higher $Z$ values $(Z>11)$.

\subsection{Beam Vertex Detector}

The beam vertex detector was recently brought into operation and used for the first ime in data acquisition during the E814 heavy ion run in June 90. This detector is used to track the trajectories of beam particles before the target. The knowledge of the horizontal position and angle of incidence of projectiles in the target is of importance in the study of all processes studied in the experiment.

In the case of central collisions. the detailed study of energy flow processes requires the knowledge of the position and angle of incidence of the beam particle at the target. For the 

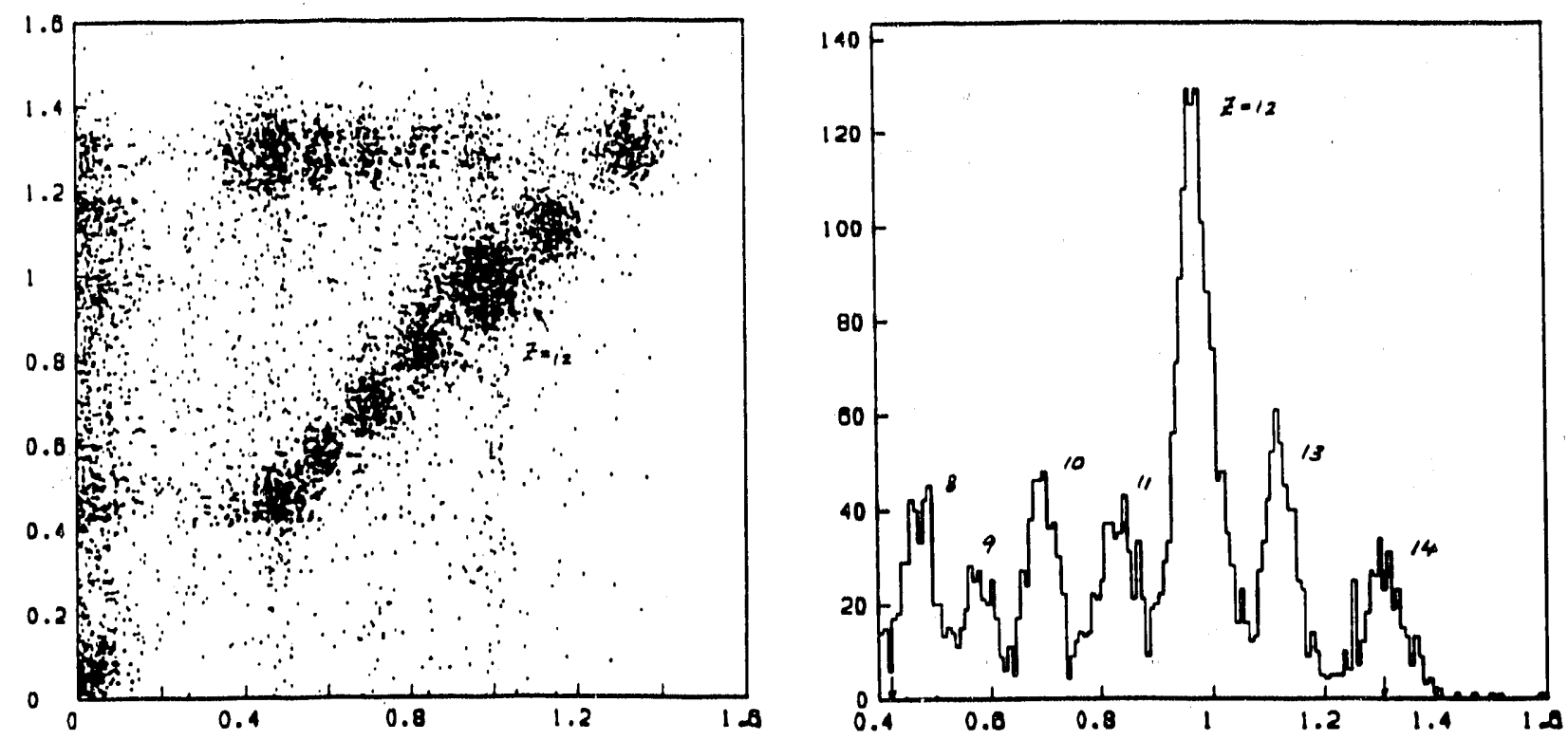

Figure 3-17: (a) Correlation between $\mathrm{Si}$ detector just after the target vs the double charge measurement (b) $Z$ spectrum after double charge measurement with combining Si detector (triple charge measurement)

study of multiplicity distributions the beam vertex information can be used to correct the rapidity values for hits in the silicon pads. In the study of peripheral processes. ther $:$ has been considerable interest [22] in the reconstruction of the spectrum of virtual photons in reactions induced by electromagnetic interactions, for which it is necessary to determine the energy transfer in the interaction. This involves measuring the difference between the energymomentum vectors of the decay products. which are measured in the forward spectrometer. and the beam particle. which is measured in the vertex detector.

In addition to its role in determining the excitation energy in peripheral interactions. the beam vertex detector is generally useful in the reconstruction of downstream tracks. For tracks exiting the target. it can be used to improve both the geometrical reconstruction and the momentum measurement by providing the $x$-position of the incident track in the target plane. The angular resolution is also improved. since the horizontal event axis is established by this measurement. Thus the momentum and angular resolution of reconstructed tracks will not depend on the horizontal size or divergence of the beam. 
$A$ drawing of the beam vertex detector and its location in the beam line is given in Figure 3-18. It consists of two silicon detectors each of which has 384 vertical strips equally spaced by 50 microns. mounted on a frame. This frame provides separate wire connestions for each strip to a small printed circuit board. which in turn is connected to the board containing the preamplifiers. The strips are back-biased PIN diodes and are thus sensitive to the passage of charged particles, which create electron-hole pairs and thus a current pulse through the diodes. A charge sensitive preamplifier drives a discriminator, followed by a latch unit which is read by the data acquisition system (see Figure 3-19).
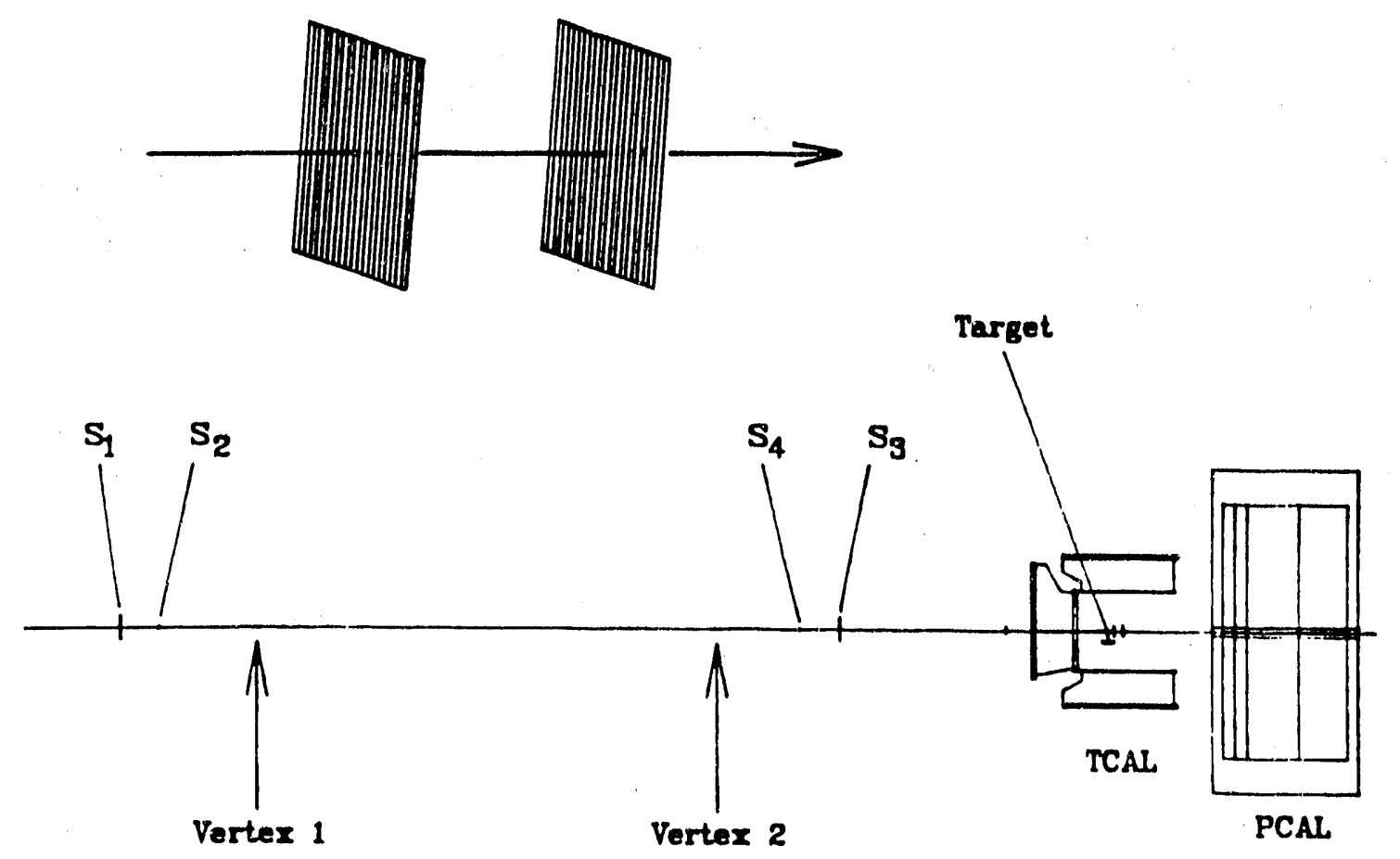

Figure 3-18: Drawing of the beam vertex detector (upper) and its location in the E814 experimental setup (lower).

Figure 3-20a shows the trajectories of beam particles for 300 events, as determined by the beam vertex detector. The $z$-coordinate is the position of the particle along the beam line. 


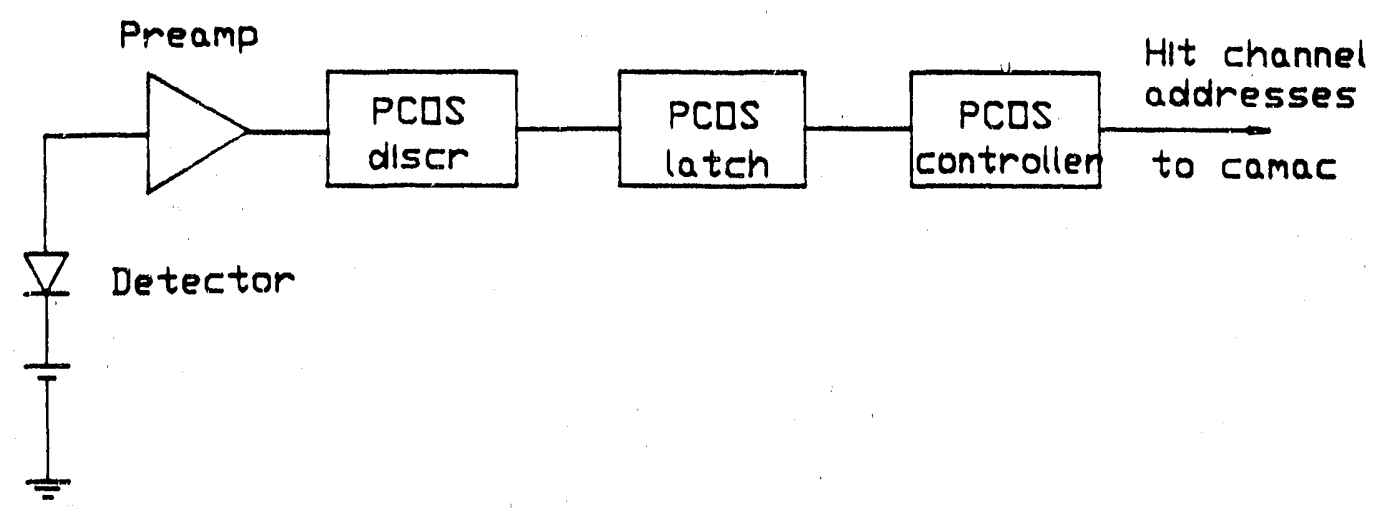

Figure 3-19: Schernatic diagram of the electronics used for the beam vertex detector. with the origin at the position of the target. The ordinate is the horizontal position perpendicular to the beam line. As can be seen. there is a variation in both the angle of the beam and position of incidence at the target. We have observed that the mean angle is dependent on the beam tuning. In the example shown, the average angle is correctly aligned with the defined beam line direction. Figure $3-20 \mathrm{~b}$ shows the dispersion of the beam horizontal position as a function of the position along the beam line.

Another use of the beam vertex detector is to provide alignment and resolution information on the drift chambers. Figure 3-21a shows the correlation between the beam track extropolated 10 $\mathrm{DCl}$ and the horizontal position of the beam measured in DC1. Figure 3-21b shows the difference between the position of the track measured in the beam vertex detector extrapolated to DC1. and the position actually measured in DC1. The resolution in this spectrum is about $0.6 \mathrm{~mm}$. which is somewhat larger than the expected value. considering effects of multiple scattering and the intrinsic resolution of the two detectors.

The vertex detector is also useful in classification of peripheral events. Figure $3-22$ a shows the correlation between the beam angle. determined by the vertex detector, and the position of the beam. measured in horizontal scintillation counter $\mathrm{H} 3$ (see Section 3.4.3). Because of the 

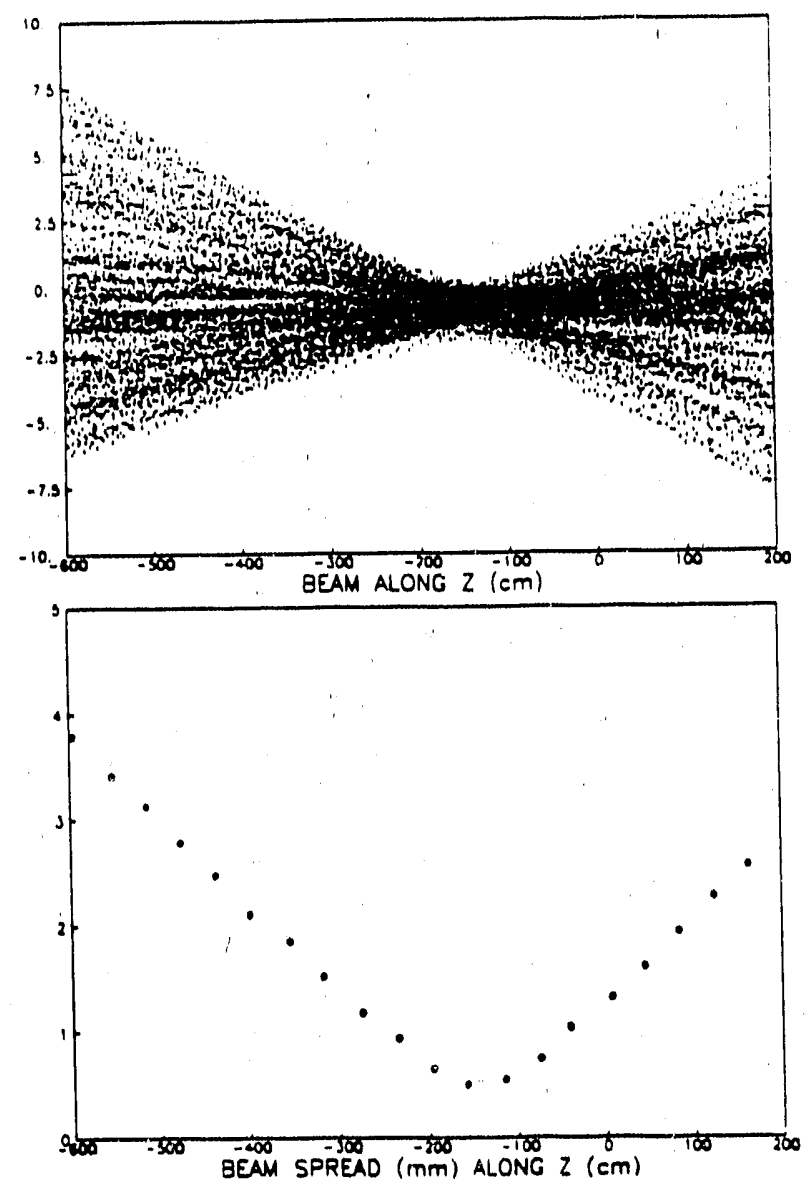

Figure 3-20: (a) Trajectorics of beam particles determined by the beam vertex system. shown in the horizontal plane.

(b) Dispersion of the beam horizontal position $(\mathrm{mm})$ as a function of the position along the beam line $(\mathrm{cm})$.

deflection in the spectrometer magnet. the position information in this counter is related to value of the $\mathrm{Z} / \mathrm{A}$ of the fragment. Because of the correlation between $\mathrm{Z} / \mathrm{A}$ and position in this counter. the position information can be used in charge identification. The position of the particles downstream are affected by the beam angle. as can be seen by the correlation exhibited in Figure 3-22a. A "raw" position spectrum. obtained by making a horizontal projection of the scatterplot in Figure 3-22a. is shown in Figure 3-22b. We apply a correction 

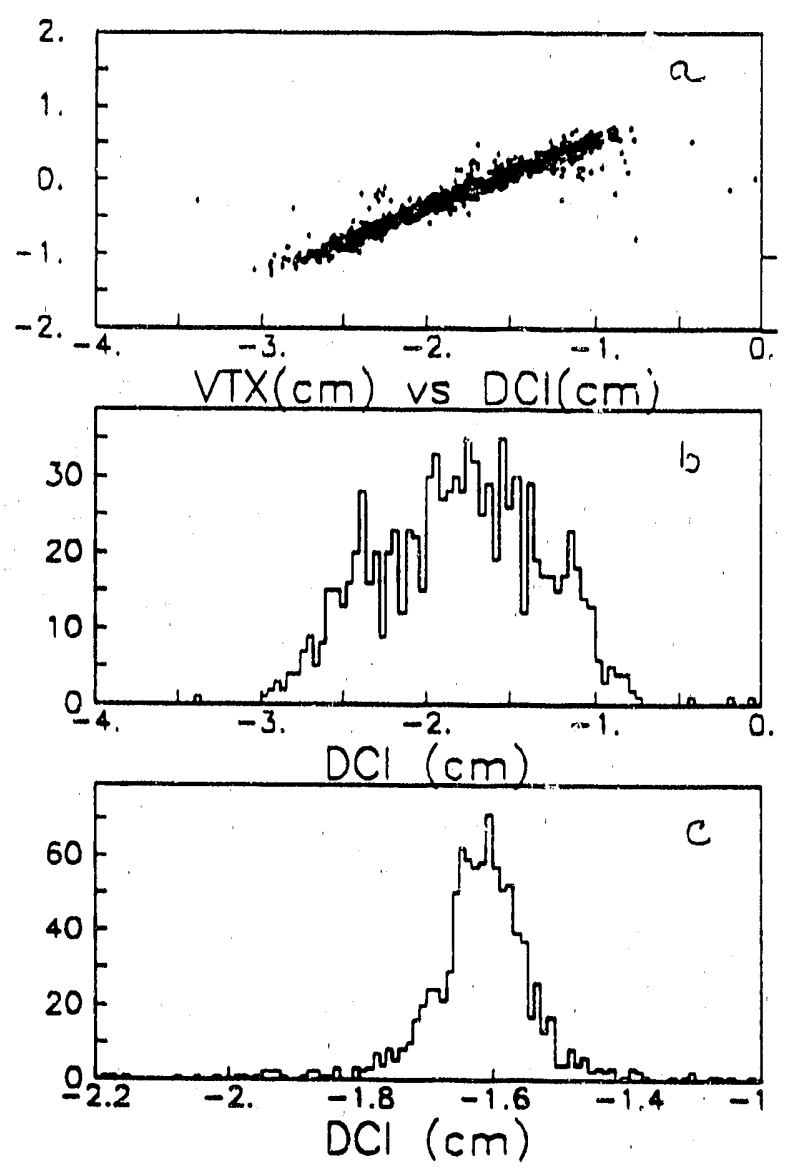

Figure 3-21: (a) beam track extroplated to DC1 versus measured position in DC1. (b) Distribution of beam particles in DCl. (c) Difference between the position measured in the beam vertex detector extrapolated to DCl. and the position measured in DCl.

based on the observed correlation to obtain the position in the counter $\mathrm{H} 3$ relative to the extrapolated beam track and hence independent of the beam angle. This position distribution is shown in Figure 3-22c, where the improvement in the resolution of the position spectrum is visible. Figure $3-23$ is a scatterplot of the $Z$ value from the triple charge measurement vs the corrected $x$ position in counter $\mathrm{H}$. The different charge states are clearly separable. 

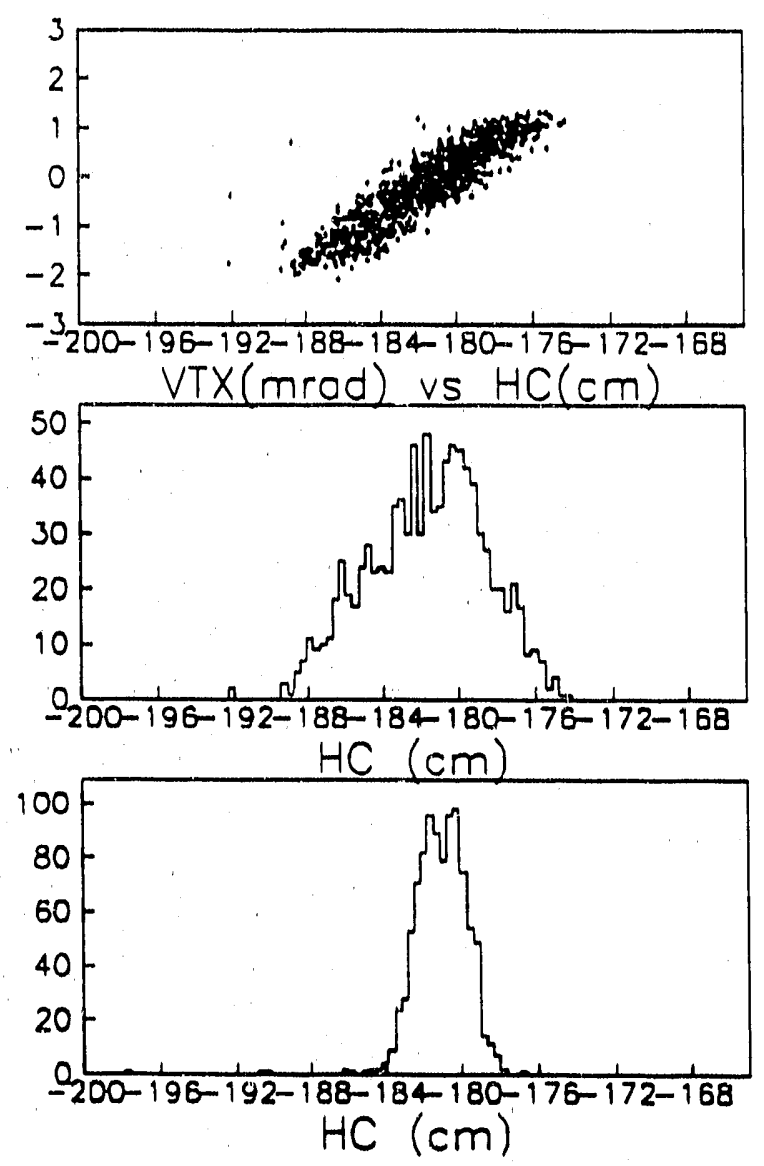

Figure 3-22: (a) Ream angle (mrad) versus beam position $(\mathrm{cm})$ at the horizontal counter H3. located behind the vertical forward scintillators. (b) Position distribution of beam particles at H3. (c) Position distribution of beam particles at $\mathrm{H} 3$. corrected to eliminate the dependence on the beam angle.

\subsection{Energy resolution in $\mathrm{Lr} / \mathrm{Cu}$ calorimeters}

We measure the energy of all the reaction products, produced by the peripheral collision which pass through a $0.8^{\circ}$ angle in the participant calorimeter by $25 \mathrm{Ur} / \mathrm{Cu}$ scintillator calorimeter modules, each segmented into several optically decoupled sections with separate wavelength shifter readouts [23]. We have three types of modules in the experiment which differ in the pattern of light decoupling cuts in the scintillator plates. One type of calorimeter (type A) has 11 horizontal light decoupling cuts in the scintillator plates separating the calorimeter into 12 towers from the bottom of the calorimeter to the top. Each tower is connected to two phototubes. The nominal fractional energy resolution of these calorimeters is 


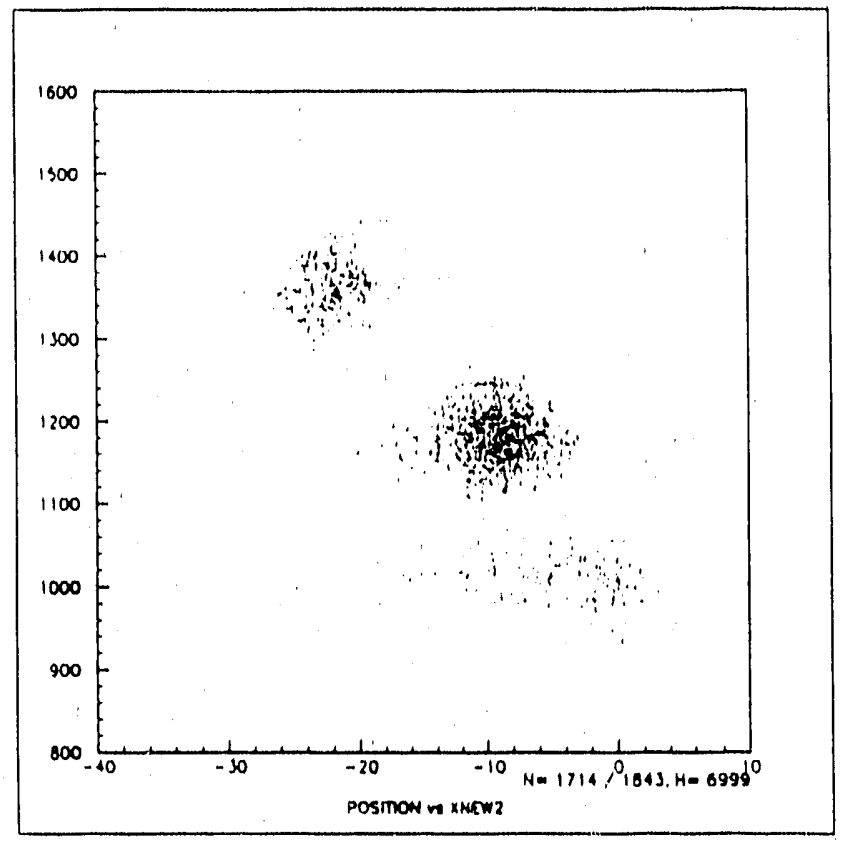

Figure 3-23: $\quad Z$ value of the triple charge measurements vs corrected position in the horizontal counter $\mathrm{H} 3$.

found 10 be $0.4 / \sqrt{ } E$. The beam calorimeter (the calorimeter where the beam hits during peripheral running) is a non-segmented calorimeter (type B). There are no light decoupling cuts in the scintillator plates. In this way it shares the large amount of light produced by the beam between its neighbors. The third type (type $C$ ) is used for the 3 upstream proton calorimeter modules. These modules have one more light decoupling cut in the scintillator plates (than in type A). which separates light sharing of all 24 phototubes from each other.

In principle we need to identify all the reaction products. The ${ }^{29} \mathrm{Si}$ beam particles should have an expected fractional energy resolution of $\sigma / E=0.4 / \sqrt{13.8 \times 28}=2 \%$. The percentage change in energy for $1 \mathrm{p}$ removal is $3.5 \%$. This means that, even with the present resolution limit it is very hard to identify a Ip removal from the pure beam. based only on the nergy. However. it was found that the measured energy resolution for "si during peripheral : unning is about 7o\%. which is a factor of 3.5 worse than the expected resolution. This makes even $2 p$ removal impossible to resolve. Understanding of this broadening is very important for the peripheral analysis. 
Figure 3-24(a) shows the beam energy as a function of the $x$ position along several calorimeter modules. This clearly shows the spatial dependence of response in the calorimeters. These calorimeters are $20 \mathrm{~cm}$ wide in the $x$ direction and the centers of the three calorimeter modules in which the beam was swept is situated roughly at $-180 \mathrm{~cm}$ (type A), $-200 \mathrm{~cm}$ (type B) and $-220 \mathrm{~cm}$ (type A). It is very clear from this plot that when the beam moves to the edge of the each calorimeter the energy is shift by $15 \%$ of its value. The $x$ position for this measurement was extracted by using the following formula,

$$
X P O S=A \log (L / R)
$$

in which $L$ and $R$ are the signals seen in the neighboring calorimeter towers and $A$ is a normalization constant extracted by verifying the correlation with the horizontal counter $x$ measurement.
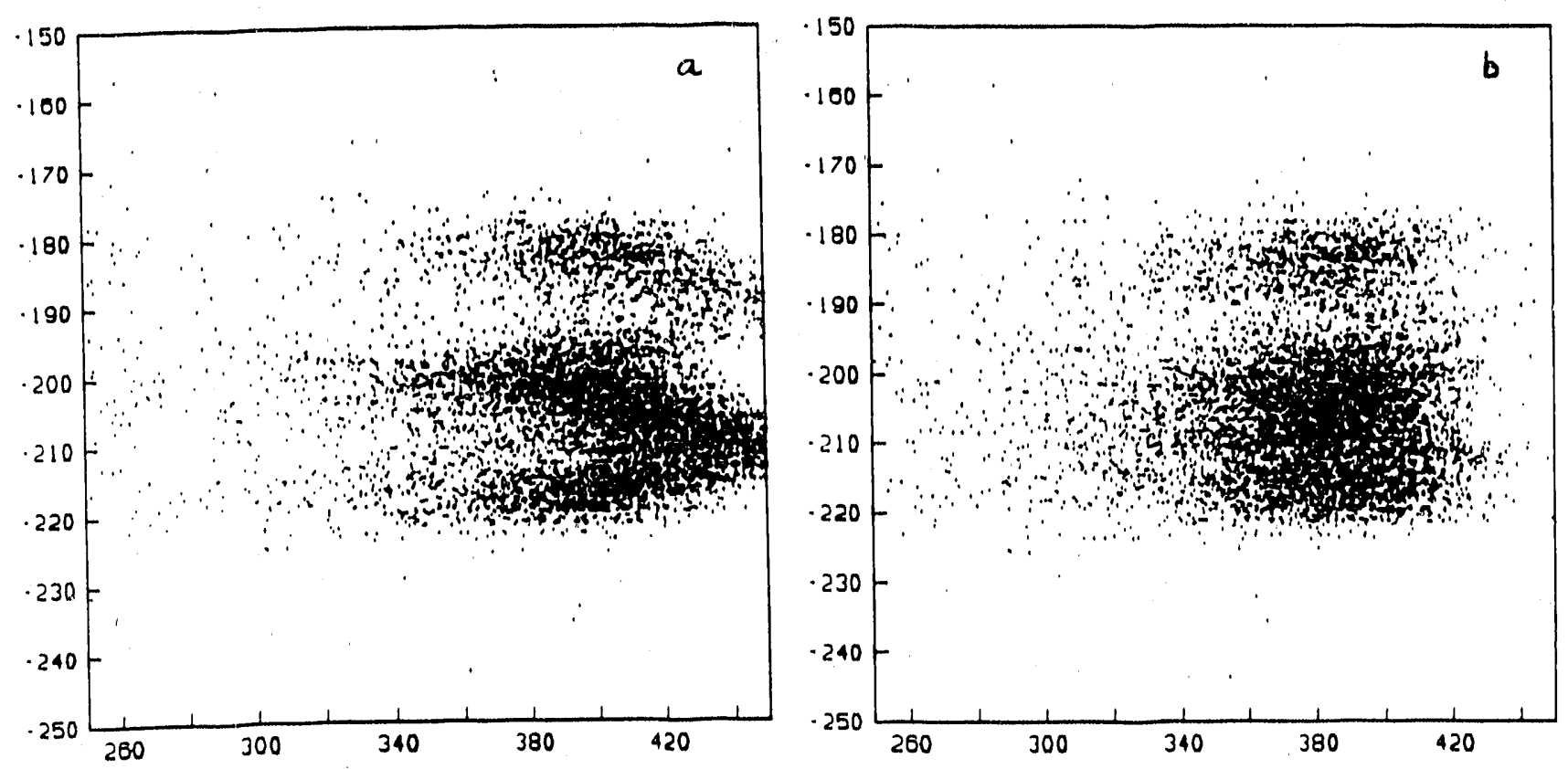

Figure 3-24: (a) Energy dependence on $x$ position. (b) Energy dependence on $x$ position after the correction. In these plots a module of type $B$ is situated at -200 $\mathrm{cm}$ and modules of rype A at $-180 \mathrm{~cm}$ and $-220 \mathrm{~cm}$.

Figure $3-24(b)$ shows the same plot after correcting for the observed $x$ dependence. We used a correction based on energy. 


$$
E_{\text {uw }}=E_{\text {.1d }}\left(1 \frac{|x P O S|}{b}\right)
$$

in which the constant $b$ was calculated assuming a linear dependence of energy on $x$. It was found that the width of the energy distribution after this correction is reduced to about $4.5 \%$. This value is still larger than the predicted resolution, but the correction depends on how well we can calculate the $x$ position on the calorimeter wall.

The same dependence was tested for the type C calorimeters, and Figure 3-25 shows the $x$ dependence of energy for the three calotimeter modules which detect protons. The energy shift here is about $25 \%$ and this must be due to the spatial nonuniformity caused by the additional vertical light decoupling cuts in these modules. Centers of the calorimeter modules are at -85 cm. $-105 \mathrm{~cm}$ and $-125 \mathrm{~cm}$.

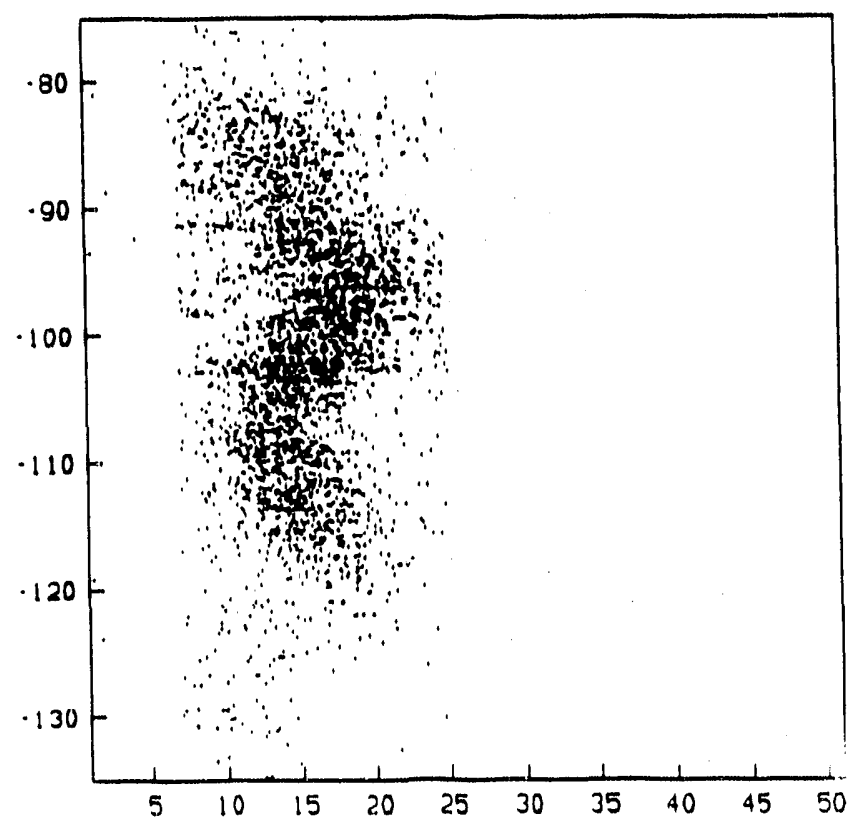

Figure 3-25: The $x$-dependence of the response of the Type $\mathrm{C}$ calorimeter.

Figure 3-26(a) shows the same plot af ter the correcting for spatial depend ce. The drop in energy at the edge of the plot clearly indicates the leakage in energy when the shower starts 
close to the edge of the module stack. We would expect about a $15 \%$ energy resolution factor for these calorimeters with intrinsic resolution of $0.4 / \sqrt{ } E$ plus fermi motion contribution. Without the $x$ position correction the observed resolution is about $22 \%$. Figure $7(b)$ shows the proton energy distribution in these modules after all corrections are made for the $x$ dependerive. The resolution factor is about 17\%, which is slightly larger than the expected value. We rely less on the calorimeter resolution in this region than in the neutron or beam region. since the proton momentum is determined through magnetic deflection. The main function of the proton calorimeters is to proved a trigger and to, aid in resolving pathological multiproton events with overlapping tracks in the drift chambers.
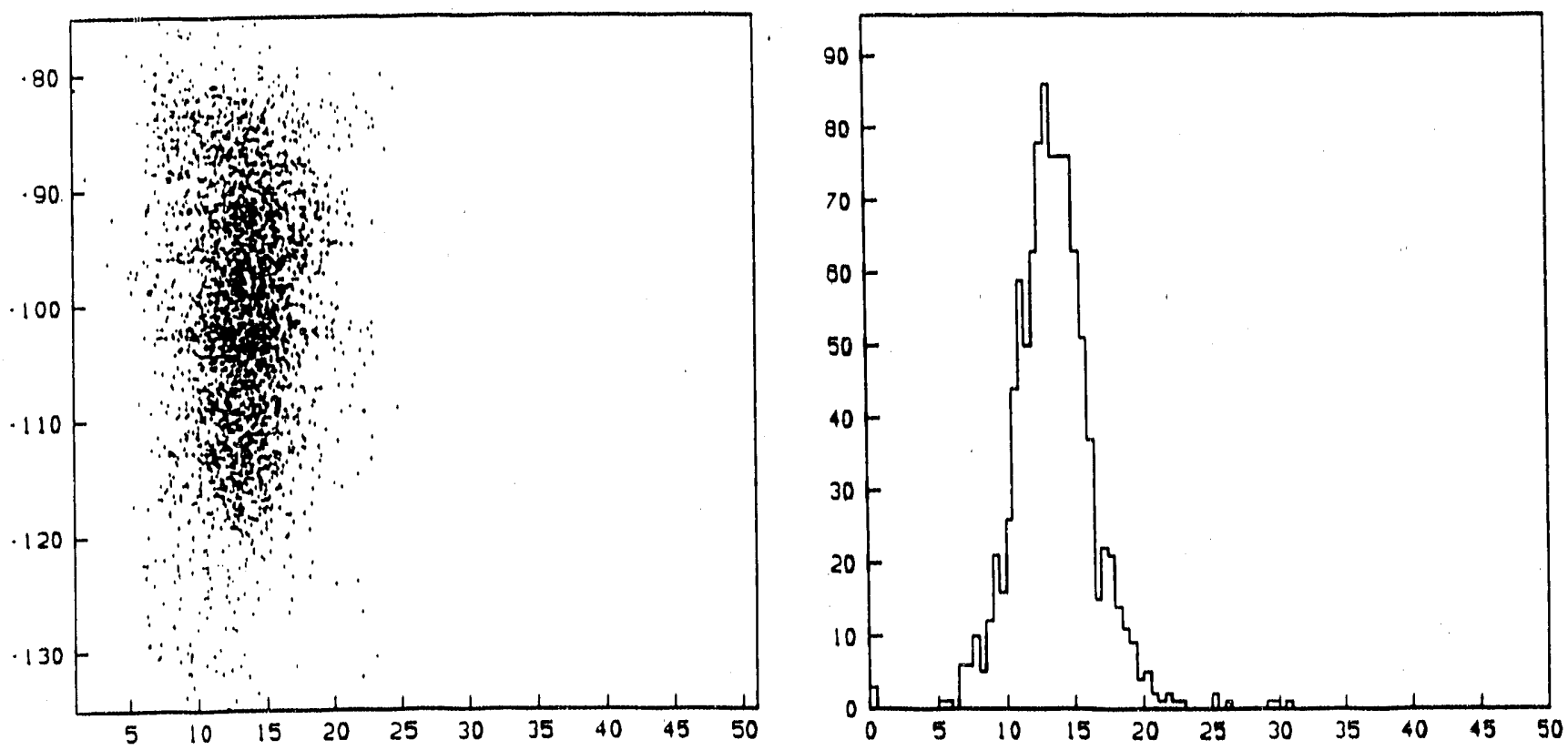

Figure 3-26: (a) $x$-dependence on proton energy after correction for spatial dependence. (b) Corrected proton energy distribution. 


\section{Publications}

During the past year, the following papers on the first results of the three physics topics of

E814 have appeared in print'. The titles, references, and abstracts of these papers are:

1. "Electromagnetic Dissociation of " ${ }^{28} \mathrm{Si}$ at $\mathrm{E}_{\mathrm{L}+\mathrm{B}} / \mathrm{A}=14.6 \mathrm{GeV}$ by Nucleon Emission." Phys, Rev C41. 1512 (1990)

Abstract: The electromagnetic dissociation cross sections for one, two and three nucleon removal were measured for an $E_{L, A B} / A=14.6 \mathrm{GeV}{ }^{28} \mathrm{Si}$ beam impinging on ${ }^{27} \mathrm{Al},{ }^{120} \mathrm{Sn}$ and ${ }^{208} \mathrm{~Pb}$ targets. The measured cross sections exhibit an approximate $\mathrm{Z}_{T}^{2}$ dependence and are well reproduced by calculations convoluting the virtual photon spectrum with the experimental $\sigma(\gamma$, nucleon) photonuclear, cross sections. Comparison with other experimental results and energy dependence of the cross sections are discussed.

2. "Energy Flow and Stopping in Relativistic Heavy-Ion Collisions at $E_{\text {liab }} / A=14.6$ GeV". Phys. Rev. Lett. 64. 1219 (1990)

Abstract: Collisions of " $\mathrm{Si}+\mathrm{Al} . \mathrm{Cu}, \mathrm{Pb}$ at $\mathrm{E}_{\mathrm{lit}, \mathrm{A}} / \mathrm{A}=14.6 \mathrm{GeV}$ were studied in a calorimetry based experiment at the BNL AGS. Transverse energy production was measured for pseudorapidities $-0.5<\pi<0.8$. Correlations with the spectra and multiplicity of neutrons and protons emitted into a forward $0.8^{\circ}$ cone demonstrate quantitatively the large amount of nuclear stopping observed in these reactions. Calculations in hadronic fireball or nucleon-nucleon based models underpredict the measured transverse energy production for $\mathrm{Si}+\mathrm{Pb}$ and indicate the need to include rescattering of secondaries and/or contributions from target fragmentation.

3. "Search for Strange Quark Matter in High Energy Heavy Ion Collisions", Physics Letters B (in press).

Abstract: We have initiated a search for strange quark matter in the products of 14.6 $\mathrm{GeV} / \mathrm{c}$ per nucleon ${ }^{28} \mathrm{Si}$ collisions with nuclei. utilizing the apparatus of E-814 at Brookhaven National Laboratory's AGS. We report the first results of this search. which is sensitive to particles with charge to mass ratios between 0.1 and 0.3 $\left(\mathrm{GeV} / \mathrm{c}^{2}\right)^{-1}$ that are produced within approximately \pm 0.5 units of the center of mass rapidity.

These papers report on results obtained from the December 1988 run. More recent data from E814 has been presented by W. Cleland [8] and T. Hemmick [10] at Quark Matter '90. 
Menton, France. There were also three presentations $[5,6,7]$ of recent results at the HIPAGS workshop held at Brookhaven National Laboratory, March 5-7. 1990. 


\section{Personnel}

1. W. E. Cleland, principal investigator. Cleland's principal role in Experiment 814 has been in the area of organization of the trigger electronics. His efforts include planning of the trigger system. decisions regarding the purchase of commercial units for the trigger, supervision of the modification of existing electronics, and the design of new electronics which must be constructed. He also is responsible for the integration of the detector systems which make up the experiment. He chaired the PCAL task force, which oversaw the commissioning of this calorimeter, and is active in the recently formed PCAL detector subgroup. This is discussed in Section 3.2.

2. D. E. Kraus. Kraus is a part-time instructor at the University of Pittsburgh with considerable experience in experimental high energy physics. $\mathrm{He}$ has spent approximately two-thirds of his time as a research associate for Experiment 814 . He is also contributing to the RHIC R\&D effort of T. J. Humanic, who is carrying out a study of the use of silicon drift chambers in RHIC experiments, and for this reason, his effort in E814 is now reduced to one-third time. His principal technical responsibilities in E814 over the past years have been in studies of the behavior of photomultiplier tubes in the environment of heavy ion experiments: design, construction. calibration and maintenance of the forward and magnet scintillators; and development of a DC light source for use in the participant and beam calorimeters.

3. K. Jayananda. Jayananda is a graduate student who plans to carry out a thesis in central collisions. He passed the comprehensive examination in May 1987, and joined our group shortly thereafter. His first effort in this experiment, carried out during the summer and fall of 1987, were to analyze the data for albedo yields taken during the May 1987 test run. He has since taken responsibility for the design and commissioning of the silicon multiplicity detector (see Section 3.3), and has helped to develop the summing electronics to integrate the information from the multiplicity detector into the second level trigger. He is now actively involved in the analysis of data from the multiplicity counter, and plans to augrnent this data with information from the participant calorimeter.

4. Bo Yu. Bo Yu is a graduate student who is carrying out a thesis in high energy physics in instrumentation under the supervision of V. Radeka of BNL. He passed the comprehensive examination in May 1986 and joined our group shortly thereafter. He has been responsible for the detailed design of the prototype for DC1, a novel type of chamber using interpolative readout of cathode pads. He has been heavily involved in the design and testing of the prototype chamber and had followed through with the design, lesting, and installation of the final chamber.

5. U. Sonnadara. Upul Sonnadara is a graduate student who has been working with us since the summer of 1988. He passed the comprehensive examination in May, 1989. He has helped with the assembly and calibration of the forward scintillation counters, and he has written monitor programs on the data-acquisition VAX in order to monitor their performance. He has taken on the responsibility for the calibration of the forward and magnet scintillators, and he is now studying the position 
dependence of the response of the forward calorimeters. He plans to write a thesis in the area of peripheral collisions.

6. E. Marcia Takagui. Takagui is a postdoctoral research associate who joined us in December 1989. She is s Jrking in the area of peripheral collisions and is presently concentrating on an understanding of the new beam vertex detector, as this will be a critical element in the full kinematic reconstruction of EMD events. which she plans to carry out. In addition she is system manager for our Silicon Graphics based computing system.

7. Z. Zhang. Zhang is a third year graduate student, who joined our group in April, 1989, having passed the comprehensive exam in May, 1987. Since fall, 1989, he has been working on the new trigger monitoring workstation discussed in Section 3.1, which was installed in E814 before the June 1990 run. His recent efforts are centered on the calibration and gain matching of the participant calorimeter, and a study of the triggering electronics for that device. He plans to write a thesis in the area of central collisions. concentrating on data from the participant calorimeter.

8. J. Rabel. Joe Rabel is a lechnician in the Pitt electronics shop who has spent considerable effort on the E814 electronics during the past three years. His expertise in digital logic has been invaluable in carrying out the design and testing of the CAMAC modules needed for our trigger processor. Mr. Rabel also maintains the electronics modules which are used in E814, including the large number of current monitors used for calibrating the calorimeters. 


\section{REFERENCES}

1. B. Bassalleck. et al., Z. Phys C 38.45 (1988).

2. J. Barrette, et al. Phys. Rev. Letters 64, 1219 (1990).

3. J. Barrette, et al, Phys. Rev. C 41. 1512 (1990).

4. J. Barrette. et al.. "Search for Strange Quark Matter in High Energy Heavy Ion Collisions," Physics Letters B, in press.

5. J. Hall et al., "Charged particle multiplicity in relativistic heavy-ion collisions," Proc. HIPAGS Meeting, March 5-7, Brookhaven National Laboratory, 1990, pp. 16-26.

6. B. Shivakumar et al., "Global oberservables in $\mathrm{Si}+$ Nucleus collisions at $14.6 \mathrm{GeV}$ per nucleon," Proc. HIPAGS Meeting, March 5-7, Brookhaven National Laboratory, 1990. pp. $1-15$.

7. R. Bellwied et al. "Baryon spectra in the low $p_{T}$ region." Proc. HIPAGS Meeting, March 5-7, Brookhaven National Laboratory, 1990, pp. 193-208.

8. W. E. Cleland, et al., "Forward Baryon Emission and Charged Multiplicity in Si-N Collisions." Proc. Quark Matter 1990. Menton. May 1990. Nucl. Phys. A. in print.

9. The E814 Collaboration. "Search for Strange Matter and Antinuclei in E-814." Proposal submitted to BNL, October. 1988.

10. T. Hemmick, et al., "Results on the production of exotic objects in relativistic nucleus nucleus collisions," Proc. Quark Matter 1990. Menton. May 1990. Nucl. Phys. A. in print.

1i. F. Videbaek et al., "Charged Particle Distributions in Heavy Ion Collisions at $14.6 \mathrm{GeV}$ A/c," Proc. HIPAGS Meeting, March 5-7, Brookhaven National Laboratory, 1990, pp. $38-44$.

12. W. Love et al., "AGS Silicon Gold Collisions Measured in the E-810 TPC." Proc. HIPAGS Meeting, March 5-7, Brookhaven National Laboratory. 1990. pp. 27-37.

13. T. Ariesson, et al. Nuclear Physics B333, 48 (1990).

14. R. Albrecht et al., Phys. Lett 201B. 89 (1988).

15. J. B. Costales. Lund model results at or near AGS energies. Internal report to E802 collaboration. Jan 12. 1988.

16. A. Giovannini and L. van Hove, Z. Phys. C-Particles and Fields 30. 391 (1986).

17. G. D. Badhwar. Nuclear Instruments and Methods 109. 119 (1973). 
18. J. H. Adams et al.. Nuclear Instruments and Methods 124. 551 (1975).

19. W. B. Christie et al.. Nuclear Instruments and Methods A255. 466 (1987).

20. C. L. Ruiz et al.: Nuclear Instruments and Methods 159: 55 (1979).

21. J. B. Birks. The Theory and Practice of Scintillation Counting, Pergamon, New York.1964.

22. C. A. Bertulani and G. Bauer, Physics Reports 163. 299 (1988).

23. M. Fatyga. D. Makowiecki. and W. Llope. Nucl. Inst. and Methods in Physics Research A284. 323 (1989).

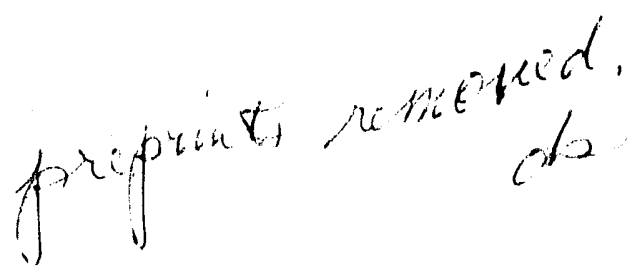



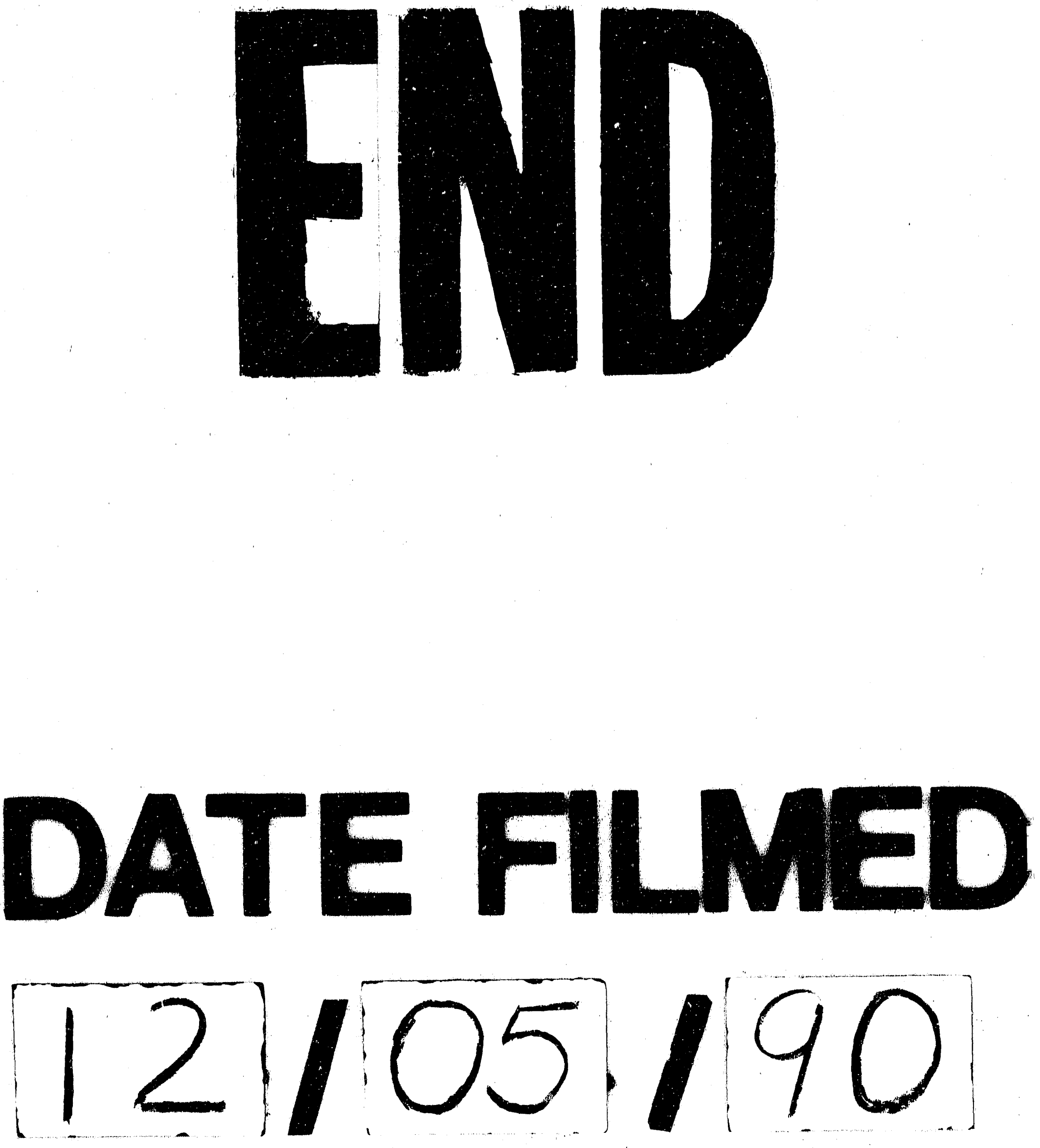
